\title{
Continuous Cohomologies of Lie Algebras of Formal $G$-Invariant Vector Fields and Obstructions to Lifting Foliations
}

By

\author{
Atsumi HAMASAKI*
}

\section{§ 0. Introduction}

In [1], I. N. Bernshtein and B. I. Rozenfeld introduced a new language which expresses differential geometric problems in terms of infinite-dimensional Lie algebras and their homogeneous spaces. In particular, their method is naturally applied to the formulation of characteristic classes of foliations. Let $a_{n}$ be the Lie algebra of all formal vector fields on $\boldsymbol{R}^{n}$. Then their results show that each element of the (continuous) cohomologies of closed transitive infinite Lie subalgebras of $\mathfrak{a}_{n}$ can be regarded as an invariant of foliations with respect to the corresponding structures such as the complex structure.

Many partial results, concerning the cohomologies of the subalgebras of $\mathfrak{a}_{n}$ such as the Lie algebra of (formal) Hamiltonian vector fields, have appeared since I. M. Gel'fand and D. B. Fuks calculated the cohomology of $\mathfrak{a}_{n}$ by the epoch-making method. But it seems that the difficulty in calculating such cohomologies hinders discovering new invariants in this approach.

In this paper, although we do not obtain any new invariants, we reveal a new relation between the classical characteristic classes (e. g. Chern classes) and the secondary classes of foliations (e.g. the Godbillon-Vey class), by using the information we obtained about the cohomologies of the Lie algebras of formal $G$-invariant vector fields $\mathfrak{a}_{n, g^{*}}$. Here $G$ is a finite dimensional Lie group with its Lie algebra $g$, and the Lie algebra of formal $G$-invariant vector fields on

Communicated by N. Shimada, April 12, 1983.

* Research Institute for Mathematical Sciences, Kyoto University, Kyoto 606, Japan. 
$\boldsymbol{R}^{n} \times G$, denoted by $\mathfrak{a}_{n, g}$, means that of the Taylor series at zero of all $G$-invariant vector fields on $\mathbb{R}^{n} \times G$ with the naturally induced bracket.

In the first half of this paper, i. e. from $\S 1$ to $\S 5$, we shall study the cohomology of $\mathfrak{a}_{n, 8}$ with the method developed by I. M. Gel'fand and D. B. Fuks in [5] (cf. [13]).

Let $\mathfrak{g l}_{n}$ denote the Lie algebra of general linear group $G L_{n}=$ $G L_{n}(\boldsymbol{R})$. We shall formulate the following theorem in $\S 2$ and prove it in $\S 3$ and $\S 4$ :

Theorem 2. 3. For any finite dimensional Lie group $G$, there exists a $G L_{n} \times G$-homomorphism $\phi$ of the truncated Weil algebra $W_{n}\left(G L_{n} \times G\right)$ of $G L_{n} \times G$ to the continuous cochain algebra of $\mathfrak{a}_{n, \mathrm{~g}}$ with trivial coefficients in $\boldsymbol{R}$ which induces an isomorphism of cohomology

$$
\phi^{*}: H^{*}\left(W_{n}\left(G L_{n} \times G\right)\right) \longrightarrow H^{*}\left(\mathfrak{a}_{n, \mathrm{~s}} ; \mathbb{R}\right) \text {. }
$$

Using Proposition 3.2 which plays an important role in proving the theorem, we obtain similar results concerning the relative cohomology with respect to subgroups of $G L_{n} \times G$ such as $H^{*}\left(\mathfrak{a}_{n, g}, O(n)\right.$; $\boldsymbol{R}) \cong H^{*}\left(W_{n}\left(G L_{n} \times G\right)_{O(n) \text {-basic }}\right)$. In $\S 5$, we give a basis of $H^{*}\left(W_{n}\left(G L_{n}\right.\right.$ $\times G)$ ) explicitly, which is similar to the "Vey basis".

The second half of this paper, i. e. from $\S 6$ to $\S 8$, is devoted to the study of one of geometric applications of the first half.

In order to use the method introduced by Bernshtein-Rozenfeld [1] and Bott-Haefliger [3], we need setting up a suitable geometric situation. Let $P$ be a smooth principal $G$-bundle and $F$ be a codimension $q$ foliation on the base space $M$ of $P$. We mean by the lifted foliation of $F$ on $P$ a $G$-invariant foliation on $P$ where each leaf is a covering space of a leaf of $F$. Assume that there exists a lifted foliation. In this situation we construct in $\S 8$ a homomorphism $\phi^{\prime}$ of $H^{*}\left(\mathfrak{a}_{q, 8}, O(q) \times G\right) \cong H^{*}\left(W_{q}\left(G L_{q} \times G\right)_{O(q) \times G \text {-basic }}\right)$ to $H^{*}(M ; \boldsymbol{R})$ by their method.

Let $\phi_{F}: H^{*}\left(W O_{q}\right) \longrightarrow H^{*}(M ; \boldsymbol{R})$ be the secondary characteristic homomorphism, and $\alpha_{P}: I(G) \longrightarrow H^{*}(M ; \boldsymbol{R})$ the Chern-Weil homomorphism for $P$ where $I(G)$ is the algebra of polynomials on $g$ invariant under conjugation by $G$. Then $H^{*}\left(W_{q}\left(G L_{q} \times G\right)_{o(q) \times G-\text { basic }}\right)$ contains the algebras, $H^{*}\left(W O_{q}\right) \cong H^{*}\left(W_{q}\left(G L_{q}\right)_{o(q) \text {-basic }}\right)$ and $I(G)$. From the naturality of the construction which is remarked in $\$ 7$, we 
shall obtain the following commutative diagram:

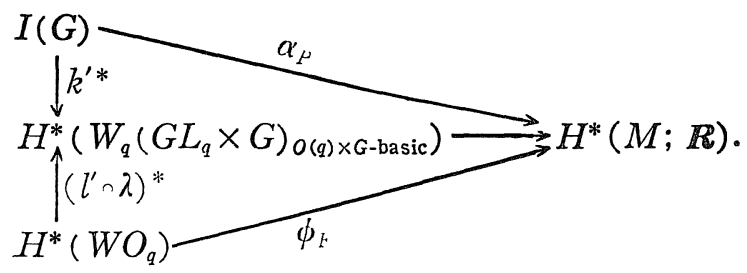

Considering the relation between $I(G)$ and $H^{*}\left(W O_{q}\right)$ in $H^{*}\left(W_{q}\left(G L_{q}\right.\right.$ $\times G)_{O(q) \times G}$ ), we shall obtain the following theorem (the statement and examples are given in $\S 6)$ :

Theorem 6. 2. Let $G$ be a Lie grous with a finite number of connected components, and $F$ be a codimension $q$ foliation on $M$, and $P$ on a smooth princifal $G$-bundle over $M$. If there exists a lifted foliation of $F$ on $P$, then for any characteristic class $\alpha_{P}(\omega)(\omega \in I(G))$ of $P$ and any secondary characteristic class $\phi_{F}^{*}\left(h_{I} c_{J}\right)$ of $F$ such that

$$
\operatorname{deg} \alpha_{P}(\omega)+\operatorname{deg} c_{J}>2 q
$$

the product

$$
\alpha_{P}(\omega) \cdot \phi_{F}^{*}\left(h_{I} c_{J}\right)=0
$$

in $H^{*}(M ; \boldsymbol{R})$. In particular, when $q=1$, the product of the GodbillonVey class and any characteristic class $\alpha_{P}(\omega)$ vanishes.

This result contains a Molino's result ([12, Proposition 1]) which says that, if the conditions of the above theorem are satisfied, then any characteristic class of $P$ whose degree is greater than $2 q$ is zero.

Other applications, for example, the relation between the cohomology of $\mathfrak{a}_{n, \mathrm{~g}}$ and the transversal foliation preserving free $G$-action, will appear elsewhere.

The author would like to express his gratitude to Professors N. Shimada and M. Adachi for their encouragement and helpful suggestion.

\section{§1. Preliminaries}

In this section we shall recall definitions of the continuous cohomology of a topological Lie algebra and of a Lie group action on a 
cochain algebra and of the Weil algebra.

First we define the continuous cohomology of a topological Lie algebra a. Let $M$ be a topological $\mathfrak{a}$-module; i. e. $M$ is a topological vector space over $\boldsymbol{R}$ and the map $\mathfrak{a} \times M \longrightarrow M$ is continuous. Let $C^{q}(\mathfrak{a} ; M)$ denote the vector space of all alternating multi-q-linear continuous maps on $\mathfrak{a}$ with values in $M$ (if $q=0, C^{0}(\mathfrak{a} ; M)=M$ ). The algebra $a$ acts on the $q$-cochain vector space $C^{q}(a ; M):$ for $X \in \mathfrak{a}$ and $\omega \in C^{q}(\mathfrak{a} ; M), X \cdot \omega=\theta(X) \omega$ is the cochain $\left(X_{1}, \cdots, X_{q}\right)$ $\longmapsto X \cdot\left(\omega\left(X_{1}, \cdots, X_{q}\right)\right)-\sum_{i=1}^{q} \omega\left(X_{1}, \cdots,\left[X, X_{i}\right], \cdots, X_{q}\right)$, and the interior product $i(X) \omega$ for $q>0$ is the cochain $\left(X_{1}, \cdots, X_{q-1}\right) \longmapsto$ $\omega\left(X, X_{1}, \cdots, X_{q-1}\right)$.

The continuous cochain space on $\mathfrak{a}$ with values in $M$ is the direct sum $C^{*}(\mathfrak{a} ; M)=\sum_{q \geq 0} C^{q}(\mathfrak{a} ; M)$ with the coboundary defined by

$$
\begin{aligned}
d \omega\left(X_{1}, \cdots, X_{q+1}\right) & =\sum_{i=1}^{q+1}(-1)^{i+1} X_{i}\left(\omega\left(X_{1}, \cdots, \hat{X}_{i}, \cdots, X_{q+1}\right)\right) \\
& +\sum_{i<j}(-1)^{i+j} \omega\left(\left[X_{i}, X_{j}\right], X_{1}, \cdots, \hat{X}_{i}, \cdots, \hat{X}_{j}, \cdots, X_{q+1}\right)
\end{aligned}
$$

for $\omega \in C^{q}(\mathfrak{a} ; M)$. The cohomology group $H^{*}(\mathfrak{a} ; M)=\sum_{q \geqq 0} H^{q}(\mathfrak{a} ; M)$ of this cochain group is called the continuous cohomology group of a with values in $M$.

When $M$ is a topological a-algebra over $\boldsymbol{R}$, we can define on $C^{*}(\mathfrak{a} ; M)$ the differential graded algebra structure by the usual shuffle product.

Remark 1. 1. With respect to operations $d, i(X)$ and $\theta(X)(X \in \mathfrak{a})$, the following relations hold (see [7, III]):

(i) $\theta(X)=i(X) d+d i(X)$

(ii) $\theta([X, Y])=\theta(X) \theta(Y)-\theta(Y) \theta(X), \quad(Y \in \mathfrak{a})$

(iii) $i([X, Y])=\theta(X) i(Y)-i(Y) \theta(X)$.

Definition 1. 2. To say that a Lie group $G$ acts on a (topological) cochain algebra $A$ means that

(i) $G$ acts smoothly on $A$ by automorphisms, in the obvious sense, and

(ii) for each $X$ in the Lie algebra $g$ of $G$, there is given an antiderivation $i(X): A \longrightarrow A$ of degree -1 such that $d i(X)+i(X) d$ $=\theta(X)$, where $\theta(X)$ is the derivation of degree 0 obtained by differentiating the $G$-action along the tangent vector $X$ at the identity, and 
(iii) $i(g \cdot X)=g \cdot i(X) \cdot g^{-1}$, where $g \cdot X$ denotes the adjoint action of $g \in G$ on $X \in \mathfrak{g}$.

We call then $A$ a $G$-cochain algebra. A $G$-homomorphism of $G$ cochain algebras is an algebra homomorphism which commutes with the derivation $i(X)$ for all $X \in \mathfrak{g}$, as well as with the elements of $G$.

Let $A$ be a $G$-cochain algebra. Then its horizontal subalgebra $A_{\text {g-horiz }}$ (also denoted by $A_{\text {horiz }}$ ) consists of the elements annihilated by $i(X)$ for all $X \in \mathfrak{g}$, and its basic subalgebra $A_{G \text {-basic }}$ (also denoted by $\left.A_{\text {basic }}\right)$ is the subalgebra of $A_{\text {horiz }}$ consisting of $G$-invariant cochains. $A_{\text {basic }}$ is also a cochain subalgebra of $A$ by Definition 1.2.

We also use the modified notion "g-cochain algebra" which can be defined without any global action. We say that a (topological) cochain algebra $A$ is a $\mathfrak{g}$-cochain algebra when for each $X \in \mathfrak{g}$, there are given an antiderivation $i(X): A \longrightarrow A$ of degree -1 and a derivation $\theta(X)$ of degree 0 satisfying two relations: (i) $\theta(X)=i(X) d$ $+d i(X)$, and (ii) $i([X, Y])=\theta(X) i(Y)-i(Y) \theta(X) \quad(Y \in \mathfrak{g})$. For example, $C^{*}(\mathfrak{a} ; \boldsymbol{R})$ is an $\mathfrak{a}$-cochain algebra. Then every $G$-cochain algebra is a $\mathfrak{g}$-cochain algebra. Replacing the $G$-action by the $\mathfrak{g}$ action, we have similar concepts with respect to $\mathfrak{g}$-cochain algebras: e. g. $A_{\mathrm{g} \text {-basic }}=\{\omega \in A ; i(X) \omega=0$, and $\theta(X) \omega=0$ for any $X \in g\}$.

Definition 1. 3. The Weil algebra $W(G)$ of $G$ is $\wedge^{*} \mathfrak{g}^{*} \otimes S^{*} \mathfrak{g}^{*}$ as algebra, where the exterior algebra $\wedge^{*} \mathrm{~g}^{*}$ is generated by 1 -forms $\alpha \in \mathfrak{g}^{*}$, and the symmetric algebra $S^{*} \mathfrak{g}^{*}$ by 2 -forms $\Omega_{\alpha}$ for $\alpha \in \mathfrak{g}^{*}$.

Its differential is defined by $d \alpha=d_{1} \alpha+\Omega_{\alpha}$, where $d_{1} \alpha \in \wedge^{2} \mathrm{~g}^{*}$ is the differential of $\alpha$ in the cochain algebra $C^{*}(g ; \boldsymbol{R})$. Its $G$-action is defined by making $i(X)$ (for $X \in \mathfrak{g}$ ) act on $\wedge^{*} \mathrm{~g}^{*}$ in the same way as $C^{*}(\mathfrak{g}: \boldsymbol{R})$, and trivially on $S^{*} \mathfrak{g}^{*}$.

Definition 1. 4. An algebraic connection for a $G$-cochain algebra $A$ is a linear map $\chi: \mathfrak{g}^{*} \longrightarrow A^{1}$ satisfying the conditions

$$
i(X) \chi(\alpha)=\alpha(X), \quad \alpha \in \mathfrak{g}^{*}, \quad X \in \mathfrak{g},
$$

and

$$
\theta(X) \cdot \chi=\chi \cdot \theta(X), \quad X \in \mathfrak{g} .
$$

In $[7$, III $]$, the following proposition is shown:

Proposition 1.5. If a G-cochain algebra $A$ has an algebraic 
connection $\chi$, then $\chi$ determines the $G$-homomorphism

$$
\chi_{w}: W(G) \longrightarrow A \text {. }
$$

\section{§ 2. The Cohomology Algebra of $\mathfrak{a}_{n, 8}$ with Values in $\boldsymbol{R}$}

In this section we give the definition of the Lie algebra $\mathfrak{a}_{n, g}$ of $G$-invariant vector fields and examine the structure of the cochain algebra $C^{*}\left(\mathfrak{a}_{n, 8} ; \boldsymbol{R}\right)$ and state the main theorem concerning the cohomology $H^{*}\left(\mathfrak{a}_{n, \mathrm{~g}} ; \boldsymbol{R}\right)$.

Let $V$ be the vector space $\boldsymbol{R}^{n}$ with a basis $\left\{\partial / \partial x^{1}, \cdots, \partial / \partial x^{n}\right\}, V^{*}$ its dual space with the dual basis $\left\{x^{1}, \cdots, x^{n}\right\}$, i. e. $\partial / \partial x^{i}\left(x^{j}\right)=\delta_{i j}$ (Kronecker's delta), and $\boldsymbol{R}[[x]]=\boldsymbol{R}\left[\left[x^{1}, \cdots, x^{n}\right]\right]=\Pi_{p \geq 0} S^{p}\left(V^{*}\right)$ the algebra of formal power series where $S^{b}$ denotes the $p$-symmetric product on $V^{*}$. Each vector $v$ of $V$ operates on $\boldsymbol{R}[[x]]$ as a derivation.

We denote by $\mathfrak{a}_{n, \mathfrak{g}}$, the tensor product $\boldsymbol{R}[[x]] \otimes(V \oplus \mathfrak{g})$ where $\mathrm{g}$ is a Lie algebra of a Lie group $G$ with a basis $\left\{H^{1}, \cdots, H^{m}\right\}, m=$ $\operatorname{dim}_{\boldsymbol{R}} \mathfrak{g}$ (from now on this basis is fixed); $\mathfrak{a}_{n, g}$ is identified with the product $\Pi_{p \geqq-1} \mathfrak{a}^{(p)}$, where $\mathfrak{a}^{(p)}=S^{p+1}\left(V^{*}\right) \otimes V \oplus S^{p}\left(V^{*}\right) \otimes \mathfrak{g} \quad\left(S^{-1}\left(V^{*}\right)=0\right)$. In particular $\mathfrak{a}^{(-1)}$ and $\mathfrak{a}^{(0)}$ are isomorphic to $V$ and $\mathfrak{g l}_{n} \times \mathfrak{g}$, respectively. Let us write $\mathfrak{g}_{n}$ for $\mathfrak{g l}_{n} \times \mathfrak{g}$.

We can naturally define on $\mathfrak{a}_{n, 8}$ the structure of Lie algebra by

$$
\left[\left(X_{1}, H_{1}\right),\left(X_{2}, H_{2}\right)\right]=\left(\left[X_{1}, X_{2}\right], X_{1} H_{2}-X_{2} H_{1}-\left[H_{1}, H_{2}\right]\right)
$$

where $X_{i}=\sum_{s=1}^{n} f_{i}^{s}(x) \partial / \partial x^{s}, \quad H_{i}=\sum_{t=1}^{m} g_{i}^{t}(x) H^{t} \quad(i=1,2)$, and $f_{i}^{s}(x)$, $g_{i}^{t}(x) \in \boldsymbol{R}[[x]]$ and

$$
\begin{aligned}
& {\left[X_{1}, X_{2}\right]=\sum_{s, t=1}^{n}\left(f_{1}^{s}(x) \partial / \partial x^{s}\left(f_{2}^{t}(x)\right)-f_{2}^{s}(x) \partial / \partial x^{s}\left(f_{1}^{t}(x)\right)\right) \partial / \partial x^{t},} \\
& {\left[X_{1}, H_{2}\right]=\sum_{s=1}^{n} \sum_{t=1}^{m} f_{1}^{s}(x) \partial / \partial x^{s}\left(g_{2}^{t}(x)\right) H^{t}} \\
& {\left[H_{1}, H_{2}\right]=\sum_{s, t, u=1}^{m} C_{s t}^{u} g_{1}^{s}(x) g_{2}^{t}(x) H^{u} .}
\end{aligned}
$$

Here $C_{s t}^{u}$ is the structure constant of $g$ with respect to the fixed basis $\left\{H^{1}, \cdots, H^{m}\right\} . \quad \mathfrak{a}_{n, 8}$ is called the Lie algebra of (right) G-invariant formal vector fields on $\boldsymbol{R}^{n} \times G$.

We can identify the Lie algebra $\mathfrak{g}_{n}$ with the subalgebra $\mathfrak{a}^{(0)}$ of $\mathfrak{a}_{n, 8}$ by the algebra homomorphism

$$
\left(a_{j}^{i}\right)_{i, j=1, \cdots, n}+H \longmapsto-\left(\sum_{i, j=1}^{n} a_{j}^{i} x^{j} \partial / \partial x^{i}+H\right)
$$

where $\left(a^{i}{ }_{j}\right)_{i, j} \in \mathfrak{g l}_{n}$ and $H \in \mathfrak{g} . \quad Z=-\sum_{i=1}^{n} x^{i} \partial / \partial x^{i}$ is an element of $\mathfrak{a}^{(0)}$ and called the radial vector field. The subspace $\mathfrak{a}^{(p)}$ of $\mathfrak{a}_{n, 8}$ is then 
the eigenspace of the adjoint map $X \longmapsto[Z, X]\left(X \in \mathfrak{a}_{n, 8}\right)$ for the eigenvalue $-t$.

We define on $\boldsymbol{R}$ the discrete topology and on $\mathfrak{a}_{n, \mathbb{g}}$ the topology associated to the filtration $\left\{\mathfrak{a}^{p}=\Pi_{r \geqq p} \mathfrak{a}^{(r)}\right\}_{p=-1,1} \ldots$. Then $\mathfrak{a}_{n, \mathbb{g}}$ has a topological Lie algebra structure over $\mathbb{R}$.

Now we consider cochain groups of $\mathfrak{a}_{n, g^{*}}$ Let $M$ be a topological $\mathfrak{a}_{n, 8}-$ module with the discrete topology. The vector space $C^{*}\left(\mathfrak{a}_{n, \mathrm{~g}} ; M\right)$ is the set of multi-q-linear alternating maps $\omega$ of $\mathfrak{a}_{n, 8} \times \cdots \times \mathfrak{a}_{n, 9}(q$ times) to $M$. For each $\omega$, from the definition of topology of $a_{n, g}$, there is an integer $m$ such that $\omega\left(X_{1}, \cdots, X_{q}\right)=0$ if one of $X_{2}$ is contained in a component $\mathfrak{a}^{m}$ of the filtration above (see [15, Proposition 3.1]). Particularly, for the trivial $\mathfrak{a}_{n, 8}$-algebra $\mathbb{R}$, we have $C^{1}\left(\mathfrak{a}_{n, \mathbb{g}} ; \boldsymbol{R}\right)=\sum_{p \geqq-1} \mathfrak{a}_{(p)}$, where $\mathfrak{a}_{(p)}=\left(\mathfrak{a}^{(p)}\right)^{*}=S^{p+1}(V) \otimes V^{*} \oplus S^{p}(V) \otimes \mathfrak{g}^{*} ;$ and $C^{q}\left(\mathfrak{a}_{n, 8} ; \boldsymbol{R}\right)=\wedge^{q} C^{1}\left(\mathfrak{a}_{n, 8} ; \mathbb{R}\right)$ is the direct sum $\bigoplus_{p_{-1}+p_{0}+p_{1}+\cdot=q}$

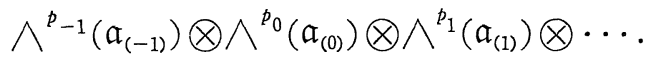

Let $\theta_{\alpha}^{2}$ and $\gamma_{\alpha}^{u}$ be the elements of $C^{1}\left(\mathfrak{a}_{n, 3} ; \mathbb{R}\right)$ where $i=1,2, \cdots, n$, $u=1,2, \cdots, m$ and $\alpha=\left(\alpha_{1}, \cdots, \alpha_{n}\right) \in Z_{0}^{n}\left(Z_{0}\right.$ : the monoid of all nonnegative integers) defined by

$$
\begin{aligned}
& \theta_{\alpha}^{i}\left(\left(\sum_{k=1}^{n} f^{k}(x) D_{k}, H\right)\right)=D^{\alpha} f^{i}(0), \\
& \gamma_{\alpha}^{u}\left(\left(X, \sum_{k=1}^{m} g^{k}(x) H^{k}\right)\right)=D^{\alpha} g^{u}(0),
\end{aligned}
$$

where $D_{s}=\partial / \partial x^{s}$ and $D^{\alpha}=D_{1}^{\alpha} \cdots D_{n}^{\alpha}$. Then $\left\{\theta_{\alpha}^{i}, \gamma_{\beta}^{u}\right\}_{i, u, \alpha, \beta}$ forms a multiplicative basis of $C^{*}\left(\mathfrak{a}_{n, 8} ; \boldsymbol{R}\right)$. In particular $\left\{\theta_{\alpha}^{i}, \gamma_{\beta}^{u} ;|\alpha|-1=\mid \beta !\right.$ $=p\}$ is a basis of $\mathfrak{a}_{(p)}$ where $|\alpha|=\alpha_{1}+\alpha_{2}+\cdots+\alpha_{n}$.

With respect to this basis, the boundary operation is expressed as follows:

$$
\begin{aligned}
d \theta_{\alpha}^{i}= & -\sum_{0 \leqq \beta \leqq \alpha, 1 \leqq k \leqq n}\left(\begin{array}{c}
\alpha \\
\beta
\end{array}\right) \theta_{\alpha-\beta+(k)}^{i} \wedge \theta_{\beta}^{k} \\
d \gamma_{\alpha}^{u}= & -\sum_{0 \leqq \beta \leqq \alpha, 1 \leqq k \leqq n}\left(\begin{array}{c}
\alpha \\
\beta
\end{array}\right) \gamma_{\alpha-\beta+(k)}^{u} \wedge \theta_{\beta}^{k} \\
& +\sum_{0 \leqq \beta \leqq \alpha, 1 \leqq v, w \leqq m}\left(\begin{array}{c}
\alpha \\
\beta
\end{array}\right) C_{v w}^{u} \gamma_{\beta}^{v} \wedge \gamma_{\alpha-\beta}^{w},
\end{aligned}
$$

where $\alpha \leqq \beta$ if and only if $\alpha_{i} \leqq \beta_{i}(i=1,2, \cdots, n), 0=(0, \cdots, 0)$, $(k)=(0, \cdots, 1, \cdots, 0)$ and $\left(\begin{array}{c}\alpha \\ \beta\end{array}\right)=\left(\begin{array}{c}\alpha_{1} \\ \beta_{1}\end{array}\right) \cdots\left(\begin{array}{c}\alpha_{n} \\ \beta_{n}\end{array}\right)$. In particular,

$$
\begin{aligned}
& d \theta_{(j)}^{i}=-\sum_{k=1}^{n} \theta_{(k)}^{i} \wedge \theta_{(j)}^{k}+\sum_{k=1}^{n} \theta^{k} \wedge \theta_{(k)+(j)}^{i} \\
& d \gamma^{u}=-\frac{1}{2} \sum_{v, w=1}^{m} C_{v w}^{u} \gamma^{v} \wedge \gamma^{w}+\sum_{k=1}^{n} \theta^{k} \wedge \gamma_{(k)}^{u} .
\end{aligned}
$$

By the way, the canonical continuous projection $\pi: \Pi_{p \geqq-1} \mathfrak{a}^{(p)} \longrightarrow \mathfrak{a}^{(0)}$ 
is a left inverse to the inclusion map $\mathfrak{g}_{n} \longrightarrow \mathfrak{a}_{n, 8}$ when we identify the Lie algebra $\mathfrak{g}_{n}$ with the subalgebra $\mathfrak{a}^{(0)}$.

We define the natural $G L_{n}$-actions on $V$ and $V^{*}$ by $A\left(\partial / \partial x^{j}\right)=$ $\sum_{i=1}^{n} a_{j}^{i} \partial / \partial x^{i}, \quad A\left(x^{i}\right)=\sum_{j=1}^{n} a_{j}^{i} x^{j} \quad\left(A=\left(a^{i}\right)_{i, j=1, \cdots, n}\right)$, and the $G$-action on $\mathfrak{g}$ by $X \cdot g=\operatorname{Ad}\left(g^{-1}\right)(X)$ where $\operatorname{Ad}\left(g^{-1}\right)(X)$ is the adjoint action of $g \in G$ on $X \in \mathrm{g}$. These two actions are commutative with each other and uniquely extended to the action on $a_{n, g}$, which preserve each $\mathfrak{a}^{(p)}$. By calculations, the isomorphism $\mathfrak{g}_{n} \cong \mathfrak{a}^{(0)}$ is a $G L_{n} \times G$-module homomorphism. Hence $\pi$ induces an algebraic connection $\mathfrak{g}_{n}^{*} \longrightarrow$ $C^{1}\left(\mathfrak{a}_{n, g} ; \boldsymbol{R}\right)$, which can be extended to the unique $G L_{n} \times G$-homomorphism $k(\pi): W=W\left(G L_{n} \times G\right) \longrightarrow C^{*}\left(\mathfrak{a}_{n, 8}: \boldsymbol{R}\right)$ by Proposition 1.5.

Define $\quad r_{j}^{i} \in \mathfrak{g l}_{n}^{*}$ by $r_{j}^{i}\left(\left(a_{v}^{u}\right)_{u, v}\right)=a_{j}^{i}$ for $\left(a_{v}^{u}\right)_{u, v} \in \mathfrak{g l}_{n}$ and let $R^{i}{ }_{j} \in S^{1} \mathfrak{g} \mathfrak{l}_{n}^{*}$ be the corresponding element in the Weil algebra $W$ (i.e. $\left.d r_{j}^{i}=-\sum_{k} r_{k}^{i} \wedge r^{k}{ }_{j}+R^{i}{ }_{j}\right)$. Take the dual basis $\left\{s^{u} \in \mathfrak{g}^{*} ; u=1, \ldots, m\right\}$ of $\left\{H^{u}\right\}_{u}$ and each corresponding element $S^{u} \in S^{1}\left(g^{*}\right)$ in the Weil algebra. Then from the definition and formulae $(2.1), k(\pi)$ can be expressed as follows:

$$
\begin{array}{ll}
k(\pi)\left(r_{j}^{i}\right)=-\theta_{(j)}^{i}, & k(\pi)\left(R_{j}^{i}\right)=\sum_{k=1}^{n} \theta^{k} \bigwedge \theta_{(k)+(j)}^{i}, \\
k(\pi)\left(s^{u}\right)=-\gamma^{u}, & k(\pi)\left(S^{u}\right)=\sum_{k=1}^{n} \theta^{k} \bigwedge \gamma_{(k)}^{u},
\end{array}
$$

where $\theta^{k}=\theta_{0}^{k}$. So $k(\pi)\left(S^{p} \mathfrak{g}_{n}^{*}\right)$ is in $\wedge^{p} \mathfrak{a}_{(-1)} \otimes \wedge^{p} \mathfrak{a}_{(1)}$.

Since the dimension of $a_{(-1)}$ is equal to $n$, the kernel of $k(\pi)$ contains an ideal $J$ of $W$ generated by $\sum_{p>n} S^{b} \mathfrak{g}_{n}^{*}$. This ideal is a cochain subalgebra of $W$. We denote by $W_{n}=W_{n}\left(G L_{n} \times G\right)$ the quotient cochain algebra $W / J$. Then $W_{n}$ is isomorphic to the tensor product $\wedge^{*} \mathfrak{g}_{n}^{*} \otimes S \mathfrak{g}_{n}^{*}$ where $S \mathrm{~g}_{n}^{*}$ is a quotient space $S\left(\mathrm{~g}_{n}^{*}\right) /\left(\sum_{p>n} S^{p} \mathrm{~g}_{n}^{*}\right)$, and $k(\pi)$ induces a $G L_{n} \times G$-homomorphism $\phi: W_{n} \longrightarrow C^{*}\left(\mathfrak{a}_{n, 8} ; \boldsymbol{R}\right)$. The following is one of our main results. Proof will be given in the next section.

Theorem 2. 3. For any finite dimensional Lie grous $G$, the $G L_{n} \times G$-homomorphism $\phi$ induces an isomorphism of cohomology

$$
\phi^{*}: H^{*}\left(W_{n}\left(G L_{n} \times G\right)\right) \cong H^{*}\left(\mathfrak{a}_{n, \mathrm{~g}} ; \boldsymbol{R}\right) .
$$

Since $O(n)$ is a subgroup of $G L_{n}, \phi$ may be also regarded as a $O(n)$-homomorphism. Let $\phi_{\text {basic }}$ be the restriction of $\phi$ to the $O(n)$ basic cochain subalgebra. The image of $\phi_{\text {basic }}$ is contained in the $O(n)$-basic cochain subalgebra $C^{*}\left(\mathfrak{a}_{n, \mathrm{~g}}, O(n) ; \boldsymbol{R}\right)$. 
Denote by $H^{*}\left(\mathfrak{a}_{n, g}, O(n) ; \boldsymbol{R}\right)$ its cohomology algebra.

Theorem 2. 4. For any finite dimensional Lie group $G, \phi_{\text {basic }}$ induces an isomorphism of cohomology

$$
\phi_{\text {basic }} *: H^{*}\left(W_{n}\left(G L_{n} \times G\right)_{O(n) \text {-basic }}\right) \cong H^{*}\left(\mathfrak{a}_{n, \mathrm{~g}}, O(n) ; \boldsymbol{R}\right) .
$$

Remark 2. 5. We need not assume that $g$ is finite dimensional. We shall use the fact that $C^{*}=\wedge \mathfrak{g l}_{n}^{*} \otimes C^{*}{ }_{\mathrm{g}^{\mathrm{r}}}$-horiz where $C^{*}=C^{*}\left(\mathfrak{a}_{n, 8} ; \boldsymbol{R}\right)$, and each $C_{{ }_{8} r_{n} \text {-horiz }}^{q}$ is the sum of isotypical pieces corresponding to finite dimensional irreducible $\mathfrak{g l}_{n}$-module. So the theorems are still true when $\mathfrak{g}$ is an infinite dimensional Lie algebra such as $\mathfrak{a}_{m}$, where $\phi$ is regarded as a $\mathfrak{g l}_{n} \times \mathfrak{g}$-homomorphism.

Remark 2. 6. Let $G$ be a compact connected Lie group and $P \longrightarrow M$ a smooth principal $G$-bundle over a compact smooth $n$ dimensional manifold $M$. From Theorem 2. 3, we can obtain a Bott conjecture type theorem (see [4]) about the continuous cohomology of Lie algebras $L_{P}^{G}$ of formal (right) $G$-invariant vector fields on $P$; that is, the continuous cochain algebra of $L_{P}^{G}$ with respect to $C^{\infty}$ topology has a homotopy type (over the complex numbers $\boldsymbol{C}$ ) of a space of sections of some fiber bundle $E$ over $M$. We shall construct $E$ as follows: Using Sullivan's theory [16], we can construct a simply connected space $B^{\prime}$ whose cohomology with coefficients in $\mathbb{C}$ is isomorphic to the truncated polynomial algebra $\hat{S g}_{n}^{*} \otimes C$, a $\mathbb{Q}$-localization $l: B(U(n) \times G) \longrightarrow B(U(n) \times G)_{Q}$ of the classifying space for $U(n)$ $\times G$ and a continuous map $f^{\prime}: B^{\prime} \longrightarrow B(U(n) \times G)_{Q}$ which induces the quotient map

$$
f^{*}: H^{*}\left(B(U(n) \times G)_{Q} ; C\right) \cong S \mathfrak{g}_{n}^{*} \otimes C \longrightarrow \hat{S g}_{n}^{*} \otimes C \cong H^{*}\left(B^{\prime} ; C\right) .
$$

Let $B$ be a homotopical fiber product of $f^{\prime}$ and $l$; i. e.

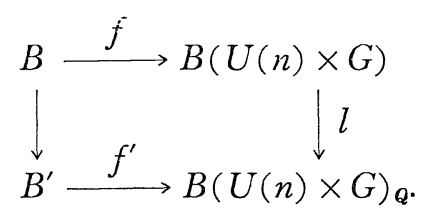

Using $f$, we have a principal $U(n) \times G$-bundle $Y$ over $B$. Then it is easy to see that the truncated Weil algebra $W_{n}\left(G L_{n} \times G\right) \otimes C$ is a model of $Y$; i. e. $H^{*}\left(W_{n}\left(G L_{n} \times G\right) ; \boldsymbol{C}\right) \cong H^{*}(Y ; \boldsymbol{C})$. Let $E^{\prime}$ be the 
fiber product $O(M) \times{ }_{M} P$ over $M$ where $O(M)$ is the orthonormal frame bundle over $M$. Let $E$ be the bundle associated with $E^{\prime}$, whose fiber is the $O(n) \times G$-space $Y$. Then using the method developed in [4], we can see that $E$ is a desired space. The details of the proof will appear elsewhere (cf. A. Hamasaki, Continuous cohomologies of Lie algebras of right $G$-invariant vecter fields on principal $G$-bundles, Master Thesis, Kyoto University, 1980).

\section{§3. Proof of Theorem 2. 3}

First we recall the decomposition of $C^{*}=C^{*}\left(\mathfrak{a}_{n, 8} ; \mathbb{R}\right)$, i. e. $C^{*}=$ $\wedge^{*} \mathfrak{a}_{(0)} \otimes\left(\otimes_{q \neq 0} \wedge^{*} \mathfrak{a}_{(q)}\right)$. Since $\mathfrak{g}_{n}^{*}=\mathfrak{a}_{(0)}, C^{*}$ is decomposed into $\wedge^{*} \mathfrak{g}_{n} \mathfrak{l}_{n}^{*}$ $\otimes B^{*}$ where $B^{*}=\wedge^{*} \mathrm{~g}^{*} \otimes\left(\otimes_{q \neq 0} \wedge^{*} \mathfrak{a}_{(q)}\right)$. From the definition it is easy to see that $B^{*}=C^{*}{ }_{8_{n}}^{*}$-horiz and $\left(\otimes_{q \neq 0} \wedge^{*} \mathfrak{a}_{(q)}\right)=C_{{ }_{g_{n}} \text {-horiz. }}^{*}$. Using these decomposition we have a descending filtration $\left\{C_{p}^{*}\right\}_{p=0,1, \ldots}$ of $C^{*}=C_{0}^{*}$ where each $C_{p}^{*}$ is the cochain subalgebra $\sum_{q \geqq p} \wedge^{*} \mathrm{~g}_{n}^{\mathrm{l}_{n}^{*}} \otimes C_{\mathrm{g}^{\mathrm{T}} n^{\mathrm{h}} \text {-horiz* }}^{q}$ It is essentially shown in [9, Theorem 1] that the associated graded differential algebra $\sum_{p \geq 0} E_{0}^{p, *}$ is isomorphic to $C^{*}\left(\mathrm{~g}_{n} ; C_{{ }^{p} r_{n}-\text { horiz }}^{p}\right)$, the cochain

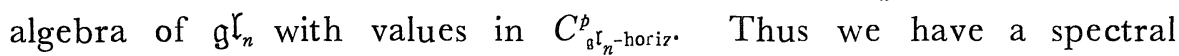
sequence

$$
E_{1}^{* * *} \cong H^{*}\left(\mathrm{~g}_{n} ; C^{*}{ }_{{ }^{1} r_{n}^{-} \text {-horiz }}\right) \Rightarrow H^{*}\left(\mathfrak{a}_{n, 8} ; \mathbb{R}\right)
$$

On the other hand the Weil algebra $W_{n}$ has a natural tensor product decomposition $\wedge^{*} \mathfrak{g}_{n}^{*} \otimes\left(\wedge^{*} \mathrm{~g}^{*} \otimes S\left(\mathfrak{g}_{n}^{*}\right)\right)$. Take a filtration $\left\{\sum_{q \geqq p} \wedge^{*} \mathfrak{g}_{n}^{\mathcal{Y}_{n}^{*}} \otimes\left(\sum_{j+2 k=q} \wedge^{j} \mathfrak{g}^{*} \otimes S^{k}\left(\mathfrak{g}_{n}^{*}\right)\right)\right\}_{p=0,1, \ldots}$. Then we have a similar spectral sequence

$$
E_{1}^{* * *} \cong H^{*}\left(\mathfrak{g}_{n} ; \wedge^{*} \mathrm{~g}^{*} \otimes S\left(\mathrm{~g}_{n}^{*}\right)\right) \Rightarrow H^{*}\left(W_{n}\right)
$$

and the homomorphism $\phi$ induces a map from the second to the first.

For a Lie algebra $\mathfrak{a}$ and a $\mathfrak{a}$-module $M$, we denote by $M^{a}$ the a-invariant submodule of $M$; i.e. $M^{\alpha}=\{m \in M ; X \cdot m=0, X \in \mathfrak{a}\}$. In order to prove that $\phi$ induces an isomorphism between the $E_{1^{-}}$ terms, we need the following two propositions:

Proposition 3.1. For a reductive Lie algebra $\mathfrak{a}$ and a finite dimensional semisimple a-module $M$, the inclusion map $M^{a} \longrightarrow M$ induces an isomorphism

$$
H^{*}(\mathfrak{a} ; \boldsymbol{R}) \otimes M^{\mathfrak{a}} \cong H^{*}(\mathfrak{a} ; M) .
$$


Proposition 3. 2. The $G L_{n} \times G$-homomorphism $\phi$ induces an isomorphism

$$
S\left(\mathrm{~g}_{n}^{*}\right)^{\mathrm{g} \mathrm{T}_{n}} \cong\left(C_{\mathrm{B}_{n}-\text { horiz }}\right)^{\mathrm{g}_{n}}
$$

where $\mathfrak{g}_{n}=\mathfrak{g l}_{n} \times \mathfrak{g}$.

Remark 3. 3. It is well-known that $S\left(\mathfrak{g l}_{n}\right)^{{ }^{\mathbb{l}_{n}}}=I\left(G L_{n}\right)$. Hence $S\left(\mathrm{~g}_{n}^{*}\right)^{{ }^{\mathrm{L}} \mathrm{l}}$ is equal to the $G L_{n}$-invariant subalgebra $S\left(\mathrm{~g}_{n}^{*}\right)^{G L_{n}}$.

The proof of Proposition 3. 1 can be found in [9, pp. 599-600] and the proof of Proposition 3. 2 will be given in the next section.

We return to the proof of Theorem 2. 3. Since $\mathrm{gl}_{n}$ is reductive (see [7]) and $\wedge^{*} \mathrm{~g}^{*}$ is a trivial $\mathrm{g}_{n}$-module, ${ }^{\prime} E_{1}^{*, *}$ is isomorphic to $H^{*}\left(\mathfrak{g l}_{n} ; \boldsymbol{R}\right) \otimes \wedge \mathfrak{g}^{*} \otimes S\left(\mathfrak{g}_{n}^{*}\right)^{\mathfrak{g}^{\mathfrak{l}}}$. On the other hand we will see in the next section that $C^{*}{ }_{{ }^{r} n_{n}}$-horiz is a sum of finite dimensional semisimple $\mathrm{gl}_{n}$-modules. By Proposition 3. 1 and the decomposition of $C^{*}{ }_{{ }^{\mathrm{r}} \mathrm{r}_{n} \text {-horizo }}$ $E_{1}^{*, *}$ is isomorphic to $H^{*}\left(\mathfrak{g l}_{n} ; \boldsymbol{R}\right) \otimes \wedge \mathfrak{g}^{*} \otimes\left(C^{*}{ }_{B_{n}}\right.$-horiz ${ }^{\mathrm{g}^{\mathrm{T}}}{ }^{\mathrm{n}}$ similarly. From Proposition 3. 2 the homomorphism $\phi$ induces an isomorphism ' $E_{1} \cong$ $E_{1}$. Hence $H^{*}\left(W_{n}\right) \cong H^{*}\left(\mathfrak{a}_{n, 8} ; \boldsymbol{R}\right)$.

The proof of Theorem 2. 4 can be done similarly (cf. [6]).

\section{§4. Proof of Proposition 3. 2}

First we shall show that $C^{*}{ }_{B_{n} \text {-horiz }}$ has a direct sum decomposition of finite dimensional semisimple $\mathfrak{g} \mathfrak{l}_{n}$-modules. Recall that each subspace $\mathfrak{a}^{(p)}$ of $\mathfrak{a}_{n, 8}$ is an eigenspace of the adjoint map of the radial vector field $Z$ for the eigenvalue $-p$. Then the dual space $\mathfrak{a}_{(p)}$ is an eigenspace of the Lie derivation $\theta(Z)$ for the eigenvalue $p$. Put for each integer

$$
U_{r}=\underset{-p_{-1}+p_{1}+2 p_{2}+\cdots+k p_{k}+\cdots=r}{\bigoplus} \wedge^{p_{-1}} \mathfrak{a}_{(-1)} \otimes \wedge^{p_{1}} \mathfrak{a}_{(1)} \otimes \wedge^{p_{2}} \mathfrak{a}_{(2)} \otimes \cdots \otimes \wedge^{p_{k}} \mathfrak{a}_{(k)} \otimes \cdots
$$

As $\operatorname{dim} \mathfrak{a}_{(-1)}$ is equal to $n, \quad C_{{ }_{\mathrm{s}_{n}} \text {-horiz }}^{*}=\bigoplus_{r \geq-n} U_{r}$. Since each $\mathfrak{a}_{(p)}$ is a finite dimensional vector space, especially $\operatorname{dim} \mathfrak{a}_{(-1)}=n, U_{r}$ is contained in the finite dimensional vector space.

$$
\sum_{-p_{-1}+p_{1}+\cdots+(r+n) p_{r+n}=r} \wedge^{p_{-1}} \mathfrak{a}_{(-1)} \otimes \wedge^{p_{1}} \mathfrak{a}_{(1)} \otimes \cdots \otimes \wedge^{p_{r+n}} \mathfrak{a}_{(r+n)} .
$$

On the other hand $U_{r}$ is an eigenspace of $\theta(Z)$ for the eigen- 
value $r$, because $\theta(Z)$ is a derivation. Since $Z$ is a generator of the center of $\mathfrak{g l}_{n}$, and from Remark 1.1 (ii), $U_{r}$ is a $\mathfrak{S l}_{n}$-module where $\mathfrak{S L}_{n}$ is the Lie algebra of the special linear group. $\mathfrak{I l}_{n}$ is simple and the direct summand of $\mathfrak{g l}_{n}$ (i.e. $\mathfrak{g l}_{n}=\mathfrak{S l}_{n} \oplus$ (center)). Hence $U_{r}$ is a finite dimensional semisimple $\mathfrak{g l}_{n}$-module. From this fact we obtain the required decomposition of $C_{B_{n} \text {-horiz }}^{*}$

Since $\phi$ can be regarded as a $\mathrm{gl}_{n}$-homomorphism, Proposition 3. 2 is a direct consequence of the following lemma:

Lemma 4. 1. (i ) $\phi\left(\hat{S}\left(\mathfrak{g}_{n}^{*}\right)^{\mathfrak{g}^{\mathfrak{l}}}\right) \supset\left(C_{\mathrm{g}_{n} \text {-horiz }}^{*}\right)^{\mathfrak{g}_{n}}$,

(ii) the restriction mat $\left.\phi\right|_{\left.\hat{s}_{\left(\mathbb{g}_{n}^{*}\right.}^{*}\right)_{n}^{\mathrm{r}}}: \hat{S}\left(\mathfrak{g}_{n}^{*}\right)^{\mathrm{g}^{\mathrm{T}}} \longrightarrow C^{*}$ is injective.

Proof of (i). From the above consideration the invariant subspace of $C^{*}$ with respect to $\theta(Z)$ is $U_{0}$. Let $T^{\otimes r}=T \otimes \cdots \otimes T$ the tensor product taken $r$ times. Since $\mathfrak{a}_{(p)}=S^{p+1}(V) \otimes V^{*} \oplus S^{p}(V) \otimes \mathfrak{g}^{*} \subset$ $V^{\otimes p+1} \otimes V^{*} \oplus V^{\otimes p} \otimes g^{*}$, we have

$$
\begin{aligned}
U_{0} \subset \sum_{-p_{-1}+p_{1}+2 p_{2}+\cdots=0} \bigotimes_{0 \leqq s_{i} \leqq p_{i}} & \left\{V^{\otimes\left(2 p_{1}-s_{1}\right)+\left(3 p_{2}-s_{2}\right)+\cdots}\right. \\
& \left.\otimes V^{* \otimes p_{-1}+\left(p_{1}-s_{1}\right)+\left(p_{2}-s_{2}\right)+\cdots} \otimes \mathfrak{g}^{* \otimes s_{1}+s_{2}+\cdots}\right\} .
\end{aligned}
$$

Let $r=p_{-1}+\left(p_{1}-s_{1}\right)+\left(p_{2}-s_{2}\right)+\cdots$ and $s=s_{1}+s_{2}+\cdots$. Then $U_{0} \subset T \equiv$ $\sum_{r, s \geq 0} V^{\otimes r} \otimes V^{* \otimes r} \otimes \mathfrak{g}^{* \otimes s} . \quad V$ can be regarded as a right $\mathfrak{g l}_{n}$-module by the operation $A \cdot v=-A v$ for $A \in g \mathfrak{l}_{n}$ and $v \in V=R^{n}$. Then the dual space of $V^{*}$ and their tensor product $V^{\otimes r} \otimes V^{* \otimes r}$ are automatically regarded as a right $\mathfrak{g l}_{n}$-module (cf. [13, Appendix]). By the easy calculation, we may consider $U_{0}$ as a $\mathrm{gl}_{n}$-submodule of $T$.

Here we apply the Weyl's invariant theory of $\mathfrak{g l}_{n}$ (see [17]).

Theorem 4. 1. Let $\left\{v_{i}\right\}$ be a basis of $V$ and $\left\{v^{i}\right\}$ be the dual basis for $V^{*}$. Then the subspace of $\mathrm{gl}_{n}$-invariants in $V^{\otimes r} \otimes V^{* \otimes r}$ is spanned by

$$
\sum_{i_{1}, \cdots, i_{r}=1}^{n} V_{i_{1}} \otimes \cdots \otimes V_{i_{r}} \otimes V^{i_{\sigma(1)}} \otimes \cdots \otimes V^{i_{\sigma(r)}}
$$

for each permutation, $\sigma$, of $1, \cdots, r$.

By the canonical projections from tensor product spaces to (anti)symmetric product spaces, the invariant basis of $V^{\otimes r} \otimes V^{* \otimes r} \otimes \mathfrak{g}^{* \otimes s}$ generates the invariant subspace $U_{0}^{\mathrm{gl}_{n}}$ which is exactly equal to

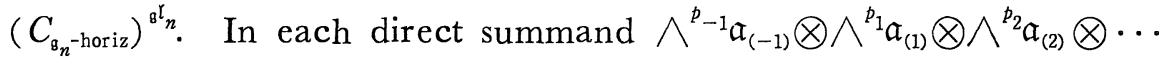


$\left(p_{-1}=p_{1}+2 p_{2}+\cdots\right)$, the invariant generators are as follows: For each permutation $\sigma$ and $0 \leqq s_{i} \leqq p_{i}$ and $1 \leqq j_{k} \leqq m\left(1 \leqq k \leqq s_{1}+s_{2}+\cdots\right)$,

$$
\begin{aligned}
& \omega_{\sigma, p, s}=\sum_{i_{1}, \cdots, i_{r}=1}^{n} \theta^{i \sigma(1)} \wedge \cdots \wedge \theta^{\left.i_{\sigma\left(p_{-1}\right)}\right)} \wedge \theta_{\left(i_{1}\right)+\left(i_{2}\right)}^{i_{\sigma\left(p_{2}+1\right)}} \wedge \cdots \wedge \theta_{\left(i_{2\left(p_{1}-s_{1}\right)-1}\right)+\left(i_{2}\left(p_{1}-s_{1}\right)\right.}^{\left.i_{\sigma\left(p_{1}\right.}+p_{1}-s_{1}\right)}
\end{aligned}
$$

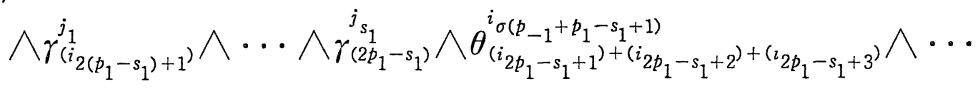

$$
\begin{aligned}
& \wedge \gamma_{\left(i_{2 p_{1}-s_{1}+3\left(p_{2}-s_{2}\right)+1}\right)+\left(i_{2 p_{1}-s_{1}+3\left(p_{2}-s_{2}\right)+2}\right)}^{j_{s_{1}+1}} \wedge \cdots \text {. }
\end{aligned}
$$

We note that $p_{-1}=p_{1}+2 p_{2}+\cdots \geqq p_{1}+p_{2}+\cdots$. If $p_{-1}>p_{1}+p_{2}+\cdots$, then there is an integer $t>1$ satisfying that $p_{t} \neq 0$, and integers $1 \leqq k_{1}$,

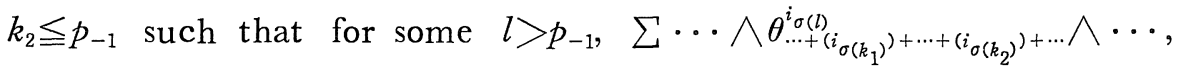

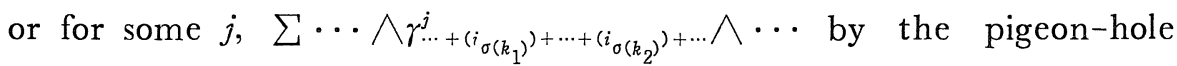
principle. Consider the first case. Put $a=i_{\sigma\left(k_{1}\right)}, b=i_{\sigma\left(k_{2}\right)}$ and $c=i_{\sigma(l)}$. Then

$$
\begin{aligned}
\pm \omega_{\sigma, p, s} & =\sum \sum_{a, b=1}^{n} \theta^{a} \wedge \theta^{b} \wedge \theta_{(a)+(b)+\cdots}^{c} \wedge \cdots \\
& =-\sum{ }_{a, b} \theta^{b} \wedge \theta^{a} \wedge \theta_{(a)+(b)+\cdots}^{c} \wedge \cdots \\
& =-\sum \sum_{d, e=1}^{n} \theta^{d} \wedge \theta^{e} \wedge \theta_{(d)+(e)+\cdots}^{c} \wedge \cdots \\
& =-\left( \pm \omega_{\sigma, p, s}\right) .
\end{aligned}
$$

Hence $\omega_{\sigma, p, s}=0$. We can do similarly in the second case. These fact shows that each non-trivial generator has a form

$$
\begin{gathered}
\sum_{\substack{i_{1}, \cdots, i_{p}=1 \\
j_{1}, \cdots, j_{p-s}=1}}^{n} \theta^{i_{1}} \wedge \cdots \wedge \theta^{i_{p}} \otimes \theta_{\left(i_{1}\right)+\left(j_{\tau(1)}\right)}^{j_{1}} \wedge \cdots \wedge \theta_{\left(i_{p-s}\right)+\left(j_{\tau(p-s)}\right)}^{j_{p-s}} \wedge \\
\otimes \gamma_{\left(i_{p-s}+1\right)}^{u_{1}} \wedge \cdots \wedge \gamma_{\left(i_{p}\right)}^{u_{s}},
\end{gathered}
$$

where $p=p_{1}, 1 \leqq s \leqq t, 1 \leqq u_{k} \leqq m(k=1,2, \cdots, s)$ and $\tau$ is a permutation of $1,2, \cdots, p-s$. From formulae 2. 2, all non-trivial generators of $\left(C_{\text {horiz }}\right)^{{ }^{\mathrm{l}_{n}}}$ are contained in the image of $\phi$. Then (i) follows immediately.

Proof of (ii). By the definition of the $\mathfrak{g l}_{n}$ action on $\mathfrak{g}_{n}$,

$$
\hat{S}\left(\mathfrak{g}_{n}^{*}\right)^{\mathbb{g}^{\mathfrak{l}}}=\sum_{p+q \leqq n} S^{p}\left(\mathfrak{g l}_{n}^{\mathfrak{l}_{n}^{*}}\right)^{\mathfrak{g}^{\mathrm{l}}} \otimes S^{q}\left(\mathfrak{g}^{*}\right) .
$$

It is well-known that $S^{*}\left(\mathrm{gl}_{n}^{*}\right)^{\mathfrak{g}^{\mathfrak{l}}}$ has a multiplicative basis $\left\{C_{1}^{\prime}, \cdots, C_{n}^{\prime}\right\}$ where $C_{k}^{\prime} \in S^{k}\left(\mathfrak{g}_{n}^{\mathfrak{l}_{n}^{*}}\right)^{\mathfrak{g}_{n}^{\mathrm{l}}} \quad(k=1,2, \cdots, n) \quad$ defined by $C_{k}^{\prime}=\operatorname{Tr} R^{k}=$ $\sum_{i_{1}, \cdots, i_{k}=1}^{n} R_{i_{2}}^{i_{1}} R_{i_{3}}^{i_{2}} \cdots R_{i_{1}}^{i_{k}}\left(R=\left(R_{j}{ }_{j}\right)_{i, j=1, \cdots, n}\right)$. Then each $\hat{S}^{p}\left(\mathrm{~g}_{n}^{*}\right)^{\mathrm{g}_{n} \mathrm{l}}(p=$ $1,2, \cdots, n)$ has an additive basis $\left\{C_{1}^{\prime q_{1}} \cdots C_{n}^{\prime q_{n}}\left(S^{1}\right)^{u_{1}} \cdots\left(S^{m}\right)^{u_{m}} ; q_{1}+2 q_{2}\right.$ 
$\left.+\cdots+n q_{n}+u_{1}+\cdots+u_{m}=p\right\}$. The image of these elements by $\phi$ are linearly independent in $C^{p}$. Indeed, if $\omega$ is a linear combination of these generators; i. e.

$$
\omega=\sum_{q_{1}+2 q_{2}+\cdots+n q_{n}+u_{1}+\cdots+u_{m}=p} a_{q_{1} \cdots q_{n}, u_{1} \cdots u_{m}} C_{1}^{\prime q_{1}} \cdots C_{n}^{\prime q_{n}} \cdot\left(S^{1}\right)^{u_{1}} \cdots\left(S^{m}\right)^{u_{m}}
$$

and $\omega=0$, then

$$
\begin{gathered}
\phi(\omega)\left[\left(\bigotimes_{i=1}^{q_{1}}\left(\partial / \partial x^{i} \otimes\left(x^{i}\right)^{2} \partial / \partial x^{i}\right)\right)\right. \\
\otimes \bigotimes_{r=2}^{n} \bigotimes_{i=1}^{q_{r}}\left(\left(\partial / \partial x^{j(r, i)+1} \otimes\left(x^{j(r, i)+1}\right)^{2} \partial / \partial x^{j(r, i+1)}\right)\right. \\
\otimes\left(\bigotimes_{k=2}^{r}\left(\partial / \partial x^{j(r, i)+k} \otimes\left(x^{j(r, i)+k}\right)^{2} \partial / \partial x^{j(r, i)+k-1}\right)\right) \\
\left.\otimes \bigotimes_{t=1}^{m} \bigotimes_{i=1}^{u_{t}}\left(\partial / \partial x^{k(t)+i} \bigotimes x^{k(t)+i} H^{t}\right)\right] \\
=q_{1} ! q_{2} ! \cdots q_{n} ! \cdot u_{1} ! \cdots u_{m} ! \cdot 2^{p} \cdot 2^{q_{2} 2^{q_{3}}} \cdots n^{q_{n}} a_{q_{1} \cdots q_{n} \cdot u_{1} \cdots u_{m}}
\end{gathered}
$$

where $j(r, i)=q_{1}+2 q_{2}+\cdots+(r-1) q_{r-1}+(i-1) r, k(t)=q_{1}+2 q_{2}+\cdots$ $+n q_{n}+u_{1}+\cdots+u_{t-1}$. Hence we obtain that $a_{q_{1} \cdots q_{n}, u_{1} \cdots u_{m}}=0$. From this (ii) follows immediately.

Remark 4.2. These dual elements were given in $[5,4.5]$. Especially

$$
\begin{aligned}
& C_{k}^{\prime}\left(\partial / \partial x^{1} \otimes\left(x^{1}\right)^{2} \partial / \partial x^{k} \otimes \partial / \partial x^{2} \otimes\left(x^{2}\right)^{2} \partial / \partial x^{1} \otimes \cdots\right. \\
& \left.\otimes \partial / \partial x^{k} \otimes\left(x^{k}\right)^{2} \partial / \partial x^{k-1}\right)=k \cdot 2^{k} .
\end{aligned}
$$

\section{§5. The "Vey Basis" of $\boldsymbol{H}^{*}\left(\mathfrak{a}_{n, 8} ; \boldsymbol{R}\right)$}

In this section we shall show the Vey basis of the cohomology of $\mathfrak{a}_{n, \mathfrak{g}}$ for reductive Lie algebras $\mathfrak{g}$. We say that a Lie algebra $\mathfrak{g}$ is reductive when its adjoint representation is semisimple. So all simple Lie algebras and all Lie algebras of compact Lie groups are reductive.

Considering Theorem 2.3, we calculate the cohomology of the truncated Weil algebra $W_{n}\left(G L_{n} \times G\right)$. To do that, the following Koszul type complexes are useful: Let $n$ and $N$ be positive integers and $V_{n}=V_{n}(N)=\oplus_{p \geq 0} V^{p}$ be a DGA (i. e. differential graded algebra) over $\boldsymbol{R}$ with the structure that

(i) as an algebra, $V=\bigwedge\left(u_{1}, \cdots, u_{N}\right) \otimes \boldsymbol{R}_{n}\left[\bar{u}_{1}, \cdots, \bar{u}_{N}\right]$ where $\wedge\left(u_{1}, \cdots, u_{N}\right)$ is an exterior algebra generated by homogeneous elements $u_{i}$, which is ordered by increasing degree, i.e. $\left|u_{i}\right| \leqq\left|u_{k}\right|$ for $i \leqq k$ whose degrees are odd; $\boldsymbol{R}_{n}\left[\bar{u}_{1}, \cdots, \bar{u}_{N}\right]$ (on homogeneous elements $\bar{u}_{i}$ of degree $\left.\left|u_{i}\right|+1\right)$ by the ideal of all elements of degree 
greater than $2 n$, and

(ii) the differential $d$ is defined by $d u_{i}=\bar{u}_{i}$ for $1 \leqq i \leqq N$, extended as an antiderivation.

Let $K$ be a subset of $\{1,2, \cdots, N\}$. We set

$$
V_{n, K}=\bigwedge_{i \in K}\left(u_{i}\right) \otimes \mathbb{R}_{n}\left[\bar{u}_{1}, \cdots, \bar{u}_{N}\right] \text {. }
$$

Then $V_{n, K}$ has a natural sub-DGA structure of $V_{n}$. Note that if $K=\{1,2, \cdots, N\}$, then $V_{n, K}=V_{n}$.

We use the following conventions: $u_{I}=u_{i_{1}} \wedge \cdots \wedge u_{i_{s}}$ for a subset $I=\left\{i_{1}, \cdots, i_{s}\right\} \subset K$, and $i_{1}<i_{2}<\cdots<i_{s} ; \bar{u}_{J}=\bar{u}_{j_{1}} \cdots \bar{u}_{j_{t}}$ for a sequence $J=\left(j_{1}, j_{2}, \cdots, j_{t}\right)$, and $1 \leqq j_{1} \leqq j_{2} \leqq \cdots \leqq j_{t} \leqq N ; i_{0}=\min \left\{i_{k} \in I\right\}$, and $j_{0}=$ $\min \left\{j_{l} \in J ; j_{l} \in K\right\} \quad(\min \phi=\infty),|J|=\left|\bar{u}_{j_{1}}\right|+\cdots+\left|\bar{u}_{j_{t}}\right|$.

Then we have the following result almost verbatim as $\mathrm{J}$. Vey has done (cf. [6, Theorem 2] and [11, Theorem 5. 110]).

Theorem 5. 1. A basis of the cohomology $H^{*}\left(V_{n, K}\right)$ is given by the classes of the monomial cocycles $u_{I} \bar{u}_{J}$ satisfying the conditions: (i) $|J| \leqq 2 n$; (ii) $i_{0} \leqq j_{0}$; (iii) if $I \neq \phi,\left|u_{i_{0}}\right|+|J| \geqq 2 n$.

Let $\boldsymbol{R}\left[c_{1}, \cdots, c_{n}\right]=S\left(\mathfrak{g l}_{n}^{*}\right)^{G L_{n}}$.

Example 5. 2. Codsider $W_{n}\left(G L_{n} \times G\right)$. Let $G$ be reductive and $\mathbb{R}\left[c_{1}^{\prime}, \cdots, c_{r}^{\prime}\right]=S(\mathfrak{g})^{G}$ where $r=\operatorname{rank} G$. Then

$$
\hat{S}\left(G L_{n} \times G\right)^{G L_{n} \times G}=\left(\mathbb{R}\left[c_{1}, \cdots, c_{n}\right] \otimes \mathbb{R}\left[c_{1}^{\prime}, \cdots, c_{r}^{\prime}\right]\right) / \text { degree }>2 n .
$$

Since all Weil algebras are acyclic, we can find $h_{i}\left(\right.$ resp. $\left.h_{i}^{\prime}\right)$ in $W_{n}\left(G L_{n} \times G\right)$ such that $d h_{i}=c_{i}\left(\right.$ resp. $\left.d h_{j}^{\prime}=c_{j}^{\prime}\right) i=1,2, \cdots, n$ (resp. $j=1,2, \cdots, r)$. Using this and by the identification $\mathbb{R}_{n}\left[u_{1}, \cdots, u_{n+r}\right]$ $=S\left(G L_{n} \times G\right)^{G L_{n}}$ we can construct a cochain map

$$
\lambda: V_{n}(n+r) \longrightarrow W_{n}\left(G L_{n} \times G\right)
$$

which induces an isomorphism in cohomology (cf. [6], [7]).

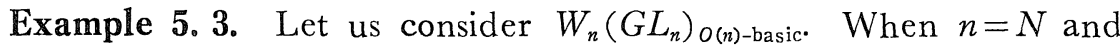
$K=\{1,3, \cdots, 2 k+1\}$ where $2 k+1$ is the largest odd integer smaller than $n, V_{n, K}$ is usually denoted by $W O_{n}$. In this case we usually write $h_{i}$ for $u_{i}$ and $c_{i}$ for $\bar{u}_{i}$. It is known that, for each $i=0,1, \cdots$, $k$, there exists $h_{2 l+1} \in W_{n}\left(\mathrm{gl}_{n}\right)_{O(n) \text {-basic }}$ such that $d h_{2 i+1}=c_{2 i+1}$, and by the identification $\mathbb{R}\left[c_{1}, \cdots, c_{n}\right]=S\left(\mathfrak{g l}_{n}^{*}\right)^{G L_{n}}$, we obtain a cochain map 


$$
\left.\lambda: W O_{n} \longrightarrow W_{n}(\mathfrak{g l})_{n}\right)_{(n) \text {-basic }}
$$

inducing an isomorphism in cohomology (see [6], [11]).

Other examples can be found in [11].

\section{§6. A Geometric Application}

From now on we consider only smooth manifolds without boundary and smooth maps.

From our definition, we note that $I(G)=S\left(\mathfrak{g}^{*}\right)^{G}$ and $I\left(G L_{n}\right)=$ $S\left(g \mathfrak{l}_{n}^{*}\right)^{G L_{n}}$.

By Formula (5.3) and Proposition 3.2, we have

$$
\left(I(G) \otimes I\left(G L_{n}\right)\right) / \mathrm{deg}>2 n \cong C_{G L_{n} \times G \text {-basic }}
$$

$I(G)$ is usually regarded as the characteristic ring of the universal principal $G$-bundle. On the other hand, let $\mathfrak{a}_{n}=\mathfrak{a}_{n,\{0\}}$ where 0 is a null Lie algebra. Note that $C^{*}=C^{*}\left(\mathfrak{a}_{n, \mathrm{~g}} ; \boldsymbol{R}\right)$ contains $C^{*}\left(\mathfrak{a}_{n} ; \boldsymbol{R}\right)$ which is used in the formulation of the secondary characteristic classes of codimension $n$ foliations. Note that $I\left(G L_{n}\right) / \mathrm{deg}>2 n$ is contained in $C^{*}\left(\mathfrak{a}_{n, 8} ; \boldsymbol{R}\right)$ and regarded as the ring of the Pontrjagin classes of normal bundles of codimension $n$ foliations. Formula (6.1) suggests a relation between them, i. e. the vanishing of their product in the higher degrees. From these studies and [12], [1], we consider a geometric significance of $C^{*}\left(\mathfrak{a}_{n, q} ; \boldsymbol{R}\right)$ in this section.

Let $\pi: P \longrightarrow M$ be a principal $G$-bundle. We consider the lifting of a codimension $q$ foliation $F$ on the base space $M$ to the total space $P$. Here we mean by the lifted foliation a $G$-invariant foliation $\bar{F}$ on $P$, each leaf of which is a covering space of a leaf of $F$ (cf. [12]).

Let $\alpha_{P}: I(G) \longrightarrow H^{*}(M ; \boldsymbol{R})$ be the Chern-Weil homomorphism for the principal $G$-bundle $P$ (cf. [7], II, p. 265). Let $\operatorname{Char}_{G} P$ denote the image of $\alpha_{P}$.

Let $\phi_{F}: H^{*}\left(W O_{q}\right) \longrightarrow H^{*}(M ; \boldsymbol{R})$ be the map which defines the secondary classes of $F([1],[3])$.

Theorem 6. 2. Let $G$ be a Lie group with a finite number of connected components and $P$ be a principal G-bundle over a manifold $M$ which has a codimension $q$ foliation. If there exists a lifted foliation of $F$ to $P$, then for any homogeneous element $\omega$ of $\operatorname{Char}_{G} P$ and 
any $h_{I} c_{J} \in H^{*}\left(W O_{q}\right)$ such that $\operatorname{deg} \omega+\operatorname{deg} c_{J}>2 q$, we have

$$
\omega \cdot \phi_{F}\left(h_{I} c_{J}\right)=0
$$

in $H^{*}(M ; \boldsymbol{R})$. In particular, when $q=1$, the product of the GodbillonVey class and any characteristic class $\omega$ vanishes.

We regard $h_{\phi} c_{\phi}$ as a unit of $H^{*}\left(W O_{q}\right)$ and $\operatorname{deg} c_{\phi}=0$, then we have the following corollary easily:

Corollary 6. 3 (cf. [12, Proposition 1]). In the same situation as above

$$
\left(\operatorname{Char}_{G} P\right)^{r}=0 \quad(r>2 q)
$$

where $\left(\operatorname{Char}_{G} P\right)^{r}=\left\{\omega \in \operatorname{Char}_{G} P ; \operatorname{deg} \omega=r\right\}$.

After we recall the characteristic homomorphism of $\Gamma$-foliation in Section 7, Theorem 6.2 will be proved in Section 8. Here we show two examples which do not satisfy the conclusion of Theorem 6.2 ; i. e. no lifted foliation exists in both cases.

Example 6. 4. Let $F$ be a foliation on a three dimensional manifold $M$ whose Godbillon-Vey invariant is non-trivial (see [2]). Let $\pi: S^{3} \longrightarrow S^{2}$ be the Hopf bundle and $\hat{p}_{i}(i=1,2)$ be projections of the product manifold $S^{2} \times M$ to the $i$-th factor. We define on $S^{2} \times M$ the induced foliation $p r_{2}^{*} F$ by $p r_{2}$ and the principal $S^{1}$-bundle induced by $p r_{1}$. Then the product of the first Chern class of $P$ and the Godbillon-Vey invariant $h_{1} c_{1}$ of $p r_{2}^{*} F$ is non-trivial.

Example 6. 5. In [10], it is shown that there exists a compact oriented manifold $M$ of dimension $n$ without boundary having a codimension $q(4 \leqq q<(n-3) / 2)$ foliation $F$ such that the rigid secondary class $h_{2} c_{q}$ is non-zero in $H^{3+2 q}(M ; \mathbb{R})$. We can assume that $n$ is odd because we may consider the product space $M \times S^{1}$ and the foliation induced by the projection of $M \times S^{1}$ to $M$. By the Poincare duality theorem, there exists an element $x$ of $H^{n-(3+2 q)}(M$; R) such that $x \cdot \phi_{F}\left(h_{2} c_{q}\right) \neq 0$. On the other hand, in view of the Chern character isomorphism from the $K$-theory with real coefficients to the ordinary real cohomology of even dimensions

$$
\operatorname{ch}: K^{0}(M) \otimes \mathbb{R} \longrightarrow H^{\text {even }}(M ; \mathbb{R}),
$$


we can find a finite number of complex vector bundles $E_{i}$ and $E_{i}^{\prime}$ over $M$ such that $\sum_{i} r_{i} \operatorname{ch}^{k}\left(E_{i}-E_{i}^{\prime}\right)=\sum_{i} r_{i}\left(\operatorname{ch}^{k}\left(E_{i}\right)-\operatorname{ch}^{k}\left(E_{i}^{\prime}\right)\right)=x$ where each $r_{i}$ is a real number and $\mathrm{ch}^{k}$ is the $k$-th Chern character $(2 k=n-(3+2 q))$. From this fact we obtain a complex vector bundle over $M$ such that $\operatorname{ch}^{k}(E) \cdot \phi_{F}\left(h_{2} c_{q}\right) \neq 0$. Let $P$ be a principal $U(n)-$ bundle associated with $E$. Then the product of the corresponding homogeneous polynomial of the Chern classes and $\phi_{F}\left(h_{2} c_{q}\right)$ is nonzero.

\section{$\S 7$. The Characteristic Homomorphisms of $\mathbb{\Gamma - F o l i a t i o n s}$}

In this section we recall the characteristic homomorphisms of $\Gamma$ foliations (see [3]).

Consider a pseudogroup $\Gamma$ whose elements are diffeomorphisms of open sets in a manifold $N$. A $\Gamma$-foliation on a smooth manifold $M$ is by definition a maximal family $F$ of submersions $f_{U}: U \longrightarrow N$ of open sets in $M$, such that for every $x \in U \cap V$ there exists an element $\gamma_{U V} \in \Gamma$ with $f_{U}=\gamma_{U V} \circ f_{V}$ in some vicinity of $x$. In this terminology the usual codimension $n$ foliation is the $\Gamma_{n}$-foliation where $\Gamma_{n}$ is the pseudogroup consisting of all local diffeomorphisms on $\boldsymbol{R}^{n}$.

The submersions $f_{U}$ are called local projections of the foliation $F$. The fiber of $f_{U}$ is a codimension $n(=\operatorname{dim} N$ ) submanifold of $U$. By $\gamma_{U V}$, these fibers are smoothly patched and define the family of connected submanifolds of $M$. Each submanifold is called the leaf of $\Gamma$-foliation. If all local projections are also immersions, we especially say that $F$ is a $\Gamma$-structure.

Given two $\Gamma$-foliations, $F$ on $M$ and $F^{\prime}$ on $M^{\prime}$, a morphism from $F$ to $F^{\prime}$ is by definition a smooth map $f: M \longrightarrow M^{\prime}$ such that for every local projection $f_{U}^{\prime} \in F^{\prime}$ the composition $f_{U}^{\prime} f: f^{-1} U \longrightarrow N$ is in $F$.

With this concept of morphism the $\Gamma$-foliations form a category $C(\Gamma)$ and we define a characteristic class of $\Gamma$-foliations with values in $\mathbb{R}$, as a natural transformation

$$
\alpha: C(\Gamma) \longrightarrow H^{*}(; \mathbb{R}) .
$$

In this paper we treat the characteristic classes in terms of Gel'fandFuks cohomology. Suppose now that $\Gamma$ is a transitive Lie-pseudogroup acting on a manifold $N$ (see [8]). Let $\mathfrak{a}(\Gamma)$ denote the Lie algebra 
of formal $\Gamma$ vector fields associated to $\Gamma$. Here a vector field defined on $U \subset N$ is called a $\Gamma$ vector field if the local onc-parameter group which it generates is in $\Gamma$, and $a(\Gamma)$ is defined as the inverse limit $\mathfrak{a}(\Gamma)=\lim \mathfrak{a}^{k}(\Gamma)$ of the $k$ jets at $o \in M$ of $\Gamma$ vector fields. Here $o$ is an arbitrary point of $M$, which will be fixed and called the origin of $M$ from now on. $a(\Gamma)$ is also a topological Lie algebra with the topology induced by the natural filtration $\mathfrak{a}(\Gamma)=F_{-1} \mathfrak{a} F_{0} \mathfrak{a} \supset F_{1} \mathfrak{a} \supset \ldots$ where $F_{k} \mathfrak{a}$ is the kernel of the projection $\mathfrak{a}(\Gamma) \longrightarrow \mathfrak{a}^{k}(\Gamma)$.

Let $\Gamma_{o}^{k}$ be the set of the $k$-jets of elements of $\Gamma$ keeping $o$ fixed. Then $\Gamma_{o}^{k}$ form an inverse system of Lie groups. It is known that there exists a subgroup $K \subset \lim \Gamma_{o}^{k}$, whose projection on every $\Gamma_{o}^{k}$ is a maximal compact subgroup for $k>0$.

Let $F$ be a foliation of $M$. Let $P^{k}(F)$ be the differentiable bundle over $M$ whose fiber over $x \in M$ is the space of $k$ jets at $x$ of local projections $f_{U}$ such that $f_{U}(x)=o$. The Lie group $\Gamma_{o}^{k}$ acts on $P^{k}(F)$ by $J_{x}^{k} f \cdot J_{o}^{k} h=J_{x}^{k}\left(h^{-1} \circ f\right)$ where $J_{x}^{k} f \in P^{k}(F)$ and $J_{o}^{k} h \in \Gamma_{o}^{k}$.

In [1] and [3], a natural injective $K$-homomorphism (also $\Gamma_{0}^{1-}$ homomorphism)

$$
\phi_{F}: C^{*}(\mathfrak{a}(\Gamma)) \longrightarrow \Omega^{*}(P(F))
$$

is constructed, where $C^{*}(\mathfrak{a}(\Gamma))$ is a continuous $K$-cochain algebra of $\mathfrak{a}(\Gamma)$ with values in $\mathbb{R}$ and $\Omega^{*}(P(F))$ is the direct limit of the de Rham complex $\Omega^{*}\left(P^{k}(F)\right)$.

Let $F$ and $F^{\prime}$ be $\Gamma$-foliations on manifolds $M$ and $M^{\prime}$ respectively, and $f: M^{\prime} \longrightarrow M$ be a morphism between them. Then $f$ induces a smooth bundle map $P^{k}(f): P^{k}\left(F^{\prime}\right) \longrightarrow P^{k}(F)$ and $P^{k}(f)^{*}: \Omega^{*}\left(P^{k}(F)\right)$ $\Omega^{*}\left(P^{k}\left(F^{\prime}\right)\right)$ is a $K$-homomorphism.

Proposition 7.2. The following diagram is commutative:

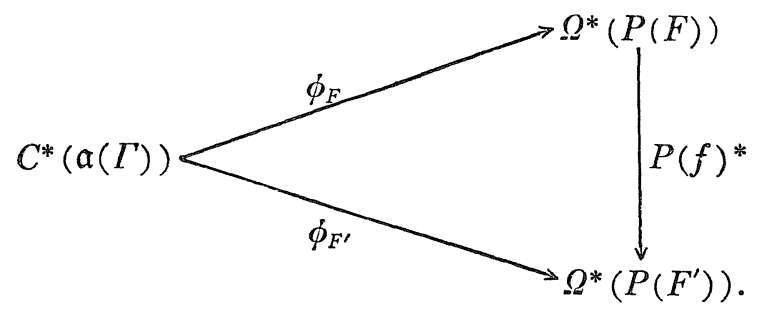

Since the $K$-basic cochain subalgebra of $\Omega^{*}\left(P^{k}(F)\right)$ is isomorphic 
to the de Rham complex of $P^{k}(F) / K$ which is a bundle over $M$ with contractible fiber $\Gamma_{o}^{k} / K, H^{*}\left(\Omega^{*}\left(P^{k}(F)\right)_{K \text {-basic }}\right)$ is isomorphic to $H^{*}(M$; $\mathbb{R})$. Using this identification, the following characteristic homomorphism is constructed in [1] and [3].

Theorem 7. 3. Let $F$ be a $\Gamma$-foliation on $M$. There is an algebra homomorphism

$$
\phi_{F}^{*}: H^{*}(\mathfrak{a}(\Gamma), K) \longrightarrow H^{*}(M ; \boldsymbol{R})
$$

which is a natural transformation on the category of $\Gamma$-foliations.

We need another fact about the naturality of $\phi_{F}$; i. e. the naturality of $\phi_{F}$ with respect to pseudogroup homomorphisms. Let $\rho: N \longrightarrow N^{\prime}$ be a submersion between smooth manifolds such that $\rho(o)=o^{\prime}$ for the origin $o$ (resp. $o^{\prime}$ ) of $N$ (resp. $N^{\prime}$ ). Let $\Gamma_{N}$ and $\Gamma_{N^{\prime}}$ be transitive Lie pseudogroups acting on $N$ and $N^{\prime}$ respectively. We say that $\rho$ induces a pseudogroup homomorphism $\rho_{*}: \Gamma_{N} \longrightarrow \Gamma_{N^{\prime}}$ when, for each local diffeomorphism $f$ in $\Gamma_{N}$ defined on the open set $U$ in $N, \rho_{*}(f)$ is a local diffeomorphism in $\Gamma_{N^{\prime}}$, defined on $\rho(U)$ such that $\rho \circ f=$ $\rho_{*}(f) \circ \rho$ (cf. [14]). Since $\rho$ can be expressed locally as a projection $\mathbb{R}^{n}=\mathbb{R}^{n^{\prime}} \times \mathbb{R}^{n-n^{\prime}} \longrightarrow \mathbb{R}^{n^{\prime}}\left(n=\operatorname{dim} N, n^{\prime}=\operatorname{dim} N^{\prime}\right)$, each $f$ in $\Gamma_{N}$ can be expressed as $f(x, y)=\left(f_{1}(x), f_{2}(x, y)\right)$ for $x \in \mathbb{R}^{n^{\prime}}, y \in \mathbb{R}^{n-n^{\prime}}$, and $\rho_{*}(f)=f_{1}$ locally. Hence $\rho$ induces a Lie group homomorphism $\rho_{\Gamma}:\left(\Gamma_{N}\right)_{o}^{k} \longrightarrow\left(\Gamma_{N^{\prime}}\right)_{o^{\prime}}^{k}$ defined by $\rho_{\Gamma}\left(J_{o}^{k} g\right)=J_{o^{\prime}}^{k} \rho_{*}(g)$ for $J_{o}^{k} g \in\left(\Gamma_{N}\right)_{o}^{k}$, and a topological Lie algebra homomorphism $\rho_{\sharp}: \mathfrak{a}\left(\Gamma_{N}\right) \longrightarrow \mathfrak{a}\left(\Gamma_{N^{\prime}}\right)$ defined by $\rho_{\#}\left(\left.J_{o}^{k} \frac{d}{d t}\right|_{t=0} g_{t}(x)\right)=\left.J_{o^{\prime}}^{k} \frac{d}{d t}\right|_{t=0} \rho_{*}\left(g_{t}\right)(x)$. Since $\rho_{\Gamma}$ is continuous, $\rho_{\Gamma}$-image of a maximal compact subgroup $K$ of $\left(\Gamma_{N}\right)_{0}^{k}(k \geqq 1)$ is contained in some maximal compact subgroup of $\left(\Gamma_{N^{\prime}}\right)_{o^{\prime}}^{k}$. We write it by $K^{\prime}$.

Let $F$ be a $\Gamma_{N}$-foliation on $M$. We mean by $\rho F$ the induced foliation $\{\rho \circ f ; f \in F\}$ on $M$. Then $\rho$ induces a smooth map $P^{k}(\rho)$ : $P^{k}(F) \longrightarrow P^{k}(\rho F)$ defined by $P^{k}(\rho)\left(J_{x}^{k} f\right)=J_{x}^{k}\left(\rho_{*} f\right)$.

By the restriction map of $\rho_{\Gamma}$ to $K, C^{*}\left(\mathfrak{a}\left(\Gamma_{N^{\prime}}\right)\right)$ and $\Omega^{*}(P(\rho F))$ are regarded as $K$-cochain algebras.

Proposition 7. 4. The following diagram is commutative: 


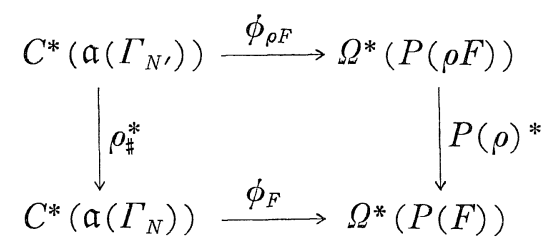

and $\rho_{\#}^{*}$ and $P(\rho) *$ are K-homomorphisms.

Proposition 7. 2 is proved in [1] and Proposition 7. 4 can be naturally obtained from the construction.

\section{§8. Proof of Theorem 6.2}

In this section we return to the special case considered in this paper. Let $G$ be a group acting on a set $S$ on the right. We say that a map $f$ of a subset $U$ to $S$ is locally G-equivariant when $f\left(x_{\tilde{B}}\right)=f(x) \tilde{z}$ for any $x \in U$ and $g \in G$ such that $x g \in U$. Let $\mathbb{R}^{q} \times G$ be a trivial principal $G$-bundle over $\mathbb{R}^{q}$. Denote by $\Gamma_{q, G}$ the pseudogroup on $\mathbb{R}^{q} \times G$ consisting of all locally $G$-equivariant local diffeomorphisms. Let $\hat{p}_{2}: \mathbb{R}^{m-q} \times\left(\mathbb{R}^{q} \times G\right) \longrightarrow \mathbb{R}^{q} \times G$ be a projection to the second factor. $\mathbb{R}^{m-q} \times \mathbb{R}^{q} \times G$ is also regarded as a trivial principal $G$-bundle over $\mathbb{R}^{m-q} \times \mathbb{R}^{q}$. Let $\Gamma_{m, q, G}$ denote the pseudogroup on $\mathbb{R}^{m-q} \times \mathbb{R}^{q} \times G$ consisting of all local diffeomorphisms $f$ for each of which there exists $\tilde{f} \in \Gamma_{q, G}$ such that $\tilde{f} \circ p_{2}=p_{2} \circ f$ on the domain of $f$. In this paper we need rather a subpseudogroup $\Gamma_{m, q, G}^{G}$ consisting of all locally $G$-equivariant elements of $\Gamma_{m, q, G}$. Note that $\Gamma_{m, n, G}^{G}=\Gamma_{m, m, G}$ $=\Gamma_{m, G}$, and $\Gamma_{m, q, G}^{G}$ is a subpseudogroup of $\Gamma_{m, G}$. It is easily seen that these pseudogroups are transitive Lie pseudogroups (cf. [8], §5).

Let $L_{g}$ be a left translation of $\mathbb{R}^{m} \times G$ defined by $L_{g}(x, h)=(x$, $g h)$ for $g \in G$ and $(x, h) \in \mathbb{R}^{m} \times G$. Then by the correspondence $g \longrightarrow L_{g}, G$ is mapped in a one-to-one fashion into $\Gamma_{m, q, G^{*}}^{G} \quad G$ acts freely on $\Gamma_{m, q, G}^{G}$ by $L_{g}^{*}(f)=f \circ L_{g}$ for $g \in G$ and $f \in \Gamma_{m, q, G}^{G}$, and also acts on $C^{*}\left(\mathfrak{a}\left(\Gamma_{m, q, G}^{G}\right)\right)$ on the right by $\left.\left(\mathbb{R}_{g} \omega\right)\left(J_{o}^{k} X\right)=\left(\left.J_{0}^{k} \frac{d}{d t}\right|_{t=0}\left(L_{g} \circ \exp t X \circ L_{g^{-1}}\right)\right\}\right)$ where $\omega \in C^{*}\left(\mathfrak{a}\left(\Gamma_{m, q, G}^{G}\right)\right)$ and $X$ is a vector field on a neighborhood of the origin $o=(0, e)$ in $\mathbb{R}^{n} \times G$ (e: the unit of $G$ ) and $\exp t X$ is a local one-parameter group generated by $X$.

Let $G L_{m, q}$ denote the subgroup of the general linear group $G L_{m}$ consisting of matrices of the form $\left[\begin{array}{cc}A & * \\ 0 & B\end{array}\right]$ where $A \in G L_{m-q}$ and 
$B \in G L_{q}$. Since any $G$-equivariant mapping of $G$ to itself preserving unit is the identity map, we can easily see that $\left(\Gamma_{m, q, G}^{G}\right)_{0}^{1}$ is the product space of $G L_{m, q}$ and $\operatorname{Hom}_{\boldsymbol{R}}\left(\boldsymbol{R}^{q}, \mathrm{~g}\right)$ which is a vector space of all $\boldsymbol{R}$ linear maps of $\boldsymbol{R}^{q}$ to a Lie algebra of $G$. Hence the maximal compact subgroup of $\left(\Gamma_{m, q, G}^{G}\right)_{o}^{k}(k>0)$ is isomorphic to $O(m-q) \times O(q)$.

Let $k$ be the dimension of $G$ and $\left\{H_{1}, \cdots, H_{k}\right\}$ a fixed basis of a Lie algebra $g$ of $G$. From the definition, it is easy to see that $\mathfrak{a}\left(\Gamma_{m, q, G}^{\mathrm{G}}\right)=\left\{X+H ; X=\sum_{j=1}^{m-q} g^{j}(y, x) \partial / \partial y^{j}+\sum_{i=1}^{q} f^{i}(x) \partial / \partial x^{i}, \quad H=\right.$ $\sum_{l=1}^{k} h^{l}(x) H^{l}, g^{j}(y, x) \in \mathbb{R}\left[\left[y^{1}, \cdots, y^{m-q}, x^{1}, \cdots, x^{q}\right]\right], f^{i}(x), h^{l}(x) \in$ $\left.R\left[\left[x^{1}, \cdots, x^{q}\right]\right]\right\}$. Hence $\mathfrak{a}\left(\Gamma_{m, q, G}^{G}\right)$ is a Lie subalgebra of $\mathfrak{a}_{m, q}$, especially $\mathfrak{a}\left(\Gamma_{m, G}\right)=\mathfrak{a}_{m, g^{*}}$ In an analogous way to the case of $\mathfrak{a}_{m, g}$, the map $X+H \longmapsto-H$ induces a $G$-cochain homomorphism

$$
\chi_{m, q, G}^{G}: W(G) \longrightarrow C^{*}\left(\mathfrak{a}\left(\Gamma_{m, q, G}^{G}\right)\right) .
$$

Recall that $\pi: P \longrightarrow M$ is the smooth $G$-bundle over the manifold with the codimension $q$ foliation and $m=\operatorname{dim} M$.

Let $\hat{p}_{1}:\left(\mathbb{R}^{m-q} \times \mathbb{R}^{q}\right) \times G \longrightarrow \boldsymbol{R}^{m-q} \times \boldsymbol{R}^{q}$ be a trivial principal $G$-bundle, and $\hat{p}_{2}: \boldsymbol{R}^{m-q} \times\left(\mathbb{R}^{q} \times G\right) \longrightarrow \boldsymbol{R}^{q} \times G$ and $\hat{p} r_{2}: \mathbb{R}^{m-q} \times \mathbb{R}^{q} \longrightarrow \mathbb{R}^{q}$ be the projections to the second factor.

Proposition 8. 2. If there is a lifted foliation $\bar{F}$ of $F$ on $P$, then for any point $x$ of $M$, there exists a neighborhood $U$ of $x$ in $M$ and a principal G-bundle isomorphism

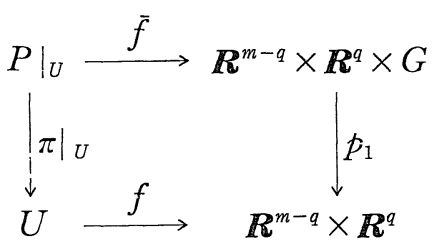

such that $p_{2} \circ \bar{f}$ is a local projection of $\bar{F}$ and $p r_{2} \circ f$ is a local projection of $F$.

Corollary 8. 3. In the same situation as above, $P$ has a $\Gamma_{m, q, G^{-}}^{G}$ structure.

Proof. Let $k=\operatorname{dim} G$. Then $\bar{F}$ is a codimension $q+k$ foliation, and for each $x \in P$, there is a neighborhood $W$ of $x$ and a diffeomorphism 
$h_{x}: W \longrightarrow \mathbb{R}^{m-q} \times \mathbb{R}^{q+k}$ such that $h_{x}(x)=0$ (origin) and the composition $p_{2}^{\prime} \circ h_{x}$, where $p_{2}^{\prime}$ is a projection from $\mathbb{R}^{m-q} \times \mathbb{R}^{q+k}$ to the second factor $\mathbb{R}^{q+k}$, is a local projection of $\bar{F}$. By the assumption we can say that for each $x \in P$, the $G$-orbit space $G \bullet x$ through $x$ and $h_{x}^{-1}\left(\mathbb{R}^{m-q} \times \mathbb{R}^{q}\right.$ $\times\{0\}$ ) intersect at $x$ transversally. Then there is a neighborhood $V$ of $o$ in $\mathbb{R}^{m-q} \times \mathbb{R}^{q}$ such that the map $k_{x}$ of $V \times G$ to the restricted bundle $\left.P\right|_{U}\left(U=\pi\left(h_{x}^{-1}(V)\right)\right)$ defined by $k_{x}(z, g)=h_{x}^{-1}(z) \cdot g$ for $z \in V$ and $g \in G$, is $G$-equivariant diffeomorphism. For $y \in \mathbb{R}^{q}$, let $V_{y}=V \cap$ $\left(\mathbb{R}^{m-q} \times\{y\}\right)$. By the definition of $h_{x}, h_{x}^{-1}\left(V_{y}\right)$ is an intersection of $\left.P\right|_{U}$ and a leaf of $F$. Because $F$ is $G$-invariant, $h_{x}^{-1}\left(V_{y}\right) \cdot g$ is also an intersection of $\left.P\right|_{U}$ and another leaf (strictly speaking, we must take the connected components of $V$ and of this intersection, respectively, because there is a foliation with a dense leaf). Hence the composition $p_{2} \circ k_{x}^{-1}:\left.P\right|_{U} \longrightarrow V \times G \longrightarrow \mathbb{R}^{m-q} \times \mathbb{R}^{q} \times G \longrightarrow \mathbb{R}^{q} \times G$ is a $G$-equivariant submersion such that for each $z \in \operatorname{Image}\left(\hat{p}_{2} \circ k_{x}^{-1}\right), k_{x} \circ p_{2}^{-1}(z)$ is an intersection of $\left.P\right|_{U}$ and a leaf of $\bar{F}$. From these facts and the definition of the lift $\bar{F}$ the proposition follows easily.

Let us denote by $\tilde{S}$ the $\Gamma_{m, q, G}^{G}$ structure on $P$. Note that $P^{0}(\tilde{S})$ $=P$ and let $r_{\tilde{S}}: P(\tilde{S}) \longrightarrow P^{0}(\tilde{S})$ be a projection. Then from (7.1) we have a sequence of cochain maps

$$
C^{*}\left(a\left(\Gamma_{m, q, G}^{G}\right)\right) \stackrel{\phi_{\tilde{S}}}{\longrightarrow} \Omega^{*}(P(\tilde{S})) \stackrel{r_{\tilde{S}}^{*}}{\longleftarrow} \Omega^{*}(P) .
$$

Note that $\phi_{\tilde{S}}$ is a $G L_{m, q}$-homomorphism.

Let $p r_{1}$ be the projection $\mathbb{R}^{m} \times G \longrightarrow \mathbb{R}^{m}$. Locally $P$ can be regarded as the trivial bundle $\mathbb{R}^{m} \times G$. From definition and Proposition 8. 2, each locally $G$-equivariant local diffeomorphism $f$ of an open set $U \subset \mathbb{R}^{m} \times G(\subset P)$ into $\mathbb{R}^{m} \times G$ can be written as $\left(f_{1}(y), f_{2}(y) h\right)$ where $(y, h) \in U, f_{1} \in \Gamma_{m}$ and $f_{2}$ is a smooth map of $V=p r_{1}(U)$ to $G$. So $f$ can be uniquely extended to a $G$-equivariant map $\hat{f}$ of $V \times G$ to $\mathbb{R}^{m} \times G$. We define a free $G$ action on $P^{k}(\tilde{S})$ by $\left(J_{x}^{k} f\right) \cdot g=J_{x g}^{k}\left(L_{g^{-1}} \circ \tilde{f}\right)$ where $x \in U$ and $g \in G$. This definition is well-defined, because $L_{g^{-1}} \circ f(y, h)=\left(f_{1}(y), g^{-1} f_{2}(y) h\right)$ for $(y, h) \in U$, and $J_{y}^{k}\left(g^{-1} f_{2}(y) g\right)$ is independent of the choice of $J_{y}^{k} f_{2}$. Hence we have a natural $G$ action on $\Omega^{*}(P(\tilde{S}))$.

Let $q: P^{k}(\tilde{S}) \longrightarrow P^{k}(\tilde{S}) / G$ be the quotient map. We write $q\left(J_{x}^{k} f\right)$ $=\left[J_{x}^{k} f\right]$. Concerning the $G$-action on $P^{k}(\tilde{S})$, we note that (i) $r_{S}^{*}$ is a $G$-homomorphism, and (ii) the quotient map $P^{k}(\tilde{S}) \longrightarrow P^{k}(\tilde{S}) / G$ 
is a local trivial bundle. The local trivialization of this bundle is given by the correspondence $J_{(y, h)}^{k} f \longmapsto\left(\left[J_{(y, h)}^{k}\left(L_{h} \circ f\right)\right], h\right)$.

Next we consider the following pseudogroups and their homomorphisms

$$
\Gamma_{m, G} \stackrel{i}{\longleftarrow} \Gamma_{m, q, G}^{G} \stackrel{p_{2 *}}{\longrightarrow} \Gamma_{q, G} \stackrel{p_{1 *}^{\prime}}{\longrightarrow} \Gamma_{q}
$$

where $i$ is an inclusion map, and $p_{2 *}$ is constructed naturally from the definition, and $p_{1 *}^{\prime}$ is a homomorphism induced by the projection $p_{1}^{\prime}: \mathbb{R}^{q} \times G \longrightarrow \mathbb{R}^{q}$.

Since all homomorphisms are induced by submersions, we can construct naturally on $P, \Gamma_{m, G^{-}}$-structure $S^{\prime}, \Gamma_{q, G}$-foliation $\bar{F}$ and $\Gamma_{q^{-}}$ foliation $F^{*}$. From Proposition 8.2 the projection induces a foliation morphism $F^{*} \longrightarrow F$. Then using the construction given in Section 7, we have a commutative diagram (8.5). From the definitions of $G^{-}$ actions on $\Gamma_{m, q, G}^{G}$ and $P(\tilde{S}), i_{\sharp}^{*}, p_{2}^{*}, P(i)^{*}$ and $P\left(p_{2}\right)^{*}$ are $G$-homomorphisms. As we have seen in Section 2, $C^{*}\left(\mathfrak{a}\left(\Gamma_{m, q, G}^{G}\right)\right)$ is generated multiplicatively by forms whose values are determined by the finite jet of formal vector fields. Hence $i_{\#}^{*}$ is surjective. It is given in $[1, \S 11]$ that $\phi_{S^{\prime}}$ is a $\mathrm{g}$-homomorphism, and by diagram chase, $\phi_{\bar{S}}$ is a $\mathfrak{g}$-homomorphism. $\phi_{\bar{F}}$ is also a $\mathfrak{g}$-homomorphism because of the following fact: $p_{2}$ has a right inverse map defined by $s(f)=\mathrm{id}_{R^{m-q}} \times f$ for $f \in \Gamma_{q, G}$, which induces a continuous homomorphism $s: \mathfrak{a}\left(\Gamma_{q, G}\right) \longrightarrow$ $\mathfrak{a}\left(\Gamma_{m, q, G}^{G}\right)$ and a smooth map $P^{k}(s): P^{k}(\bar{F}) \longrightarrow P^{k}(\tilde{S}): s_{\sharp}^{*}\left(\operatorname{resp} \cdot p(s)^{*}\right)$ is a left inverse map of $t_{2 \sharp}^{*}\left(\right.$ resp. $\left.P\left(p_{2}\right)^{*}\right)$. Therefore $p_{2 \sharp}^{*}$ and $P\left(p_{2}\right)^{*}$ are injective. By diagram chase, $\phi_{\bar{F}}$ is a $\mathrm{g}$-homomorphism.

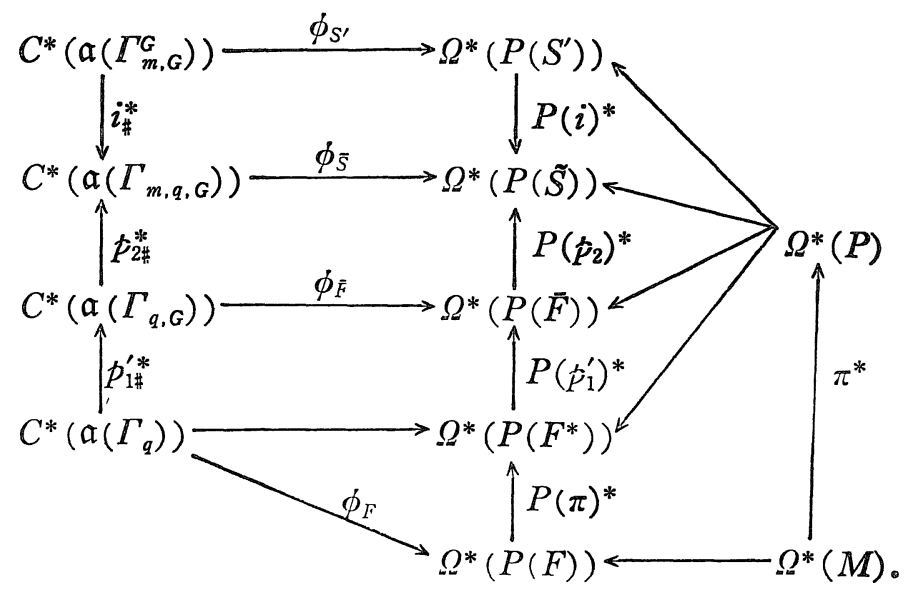


On the other hand, from (8.1) and Section 2, we obtain a commutative diagram:

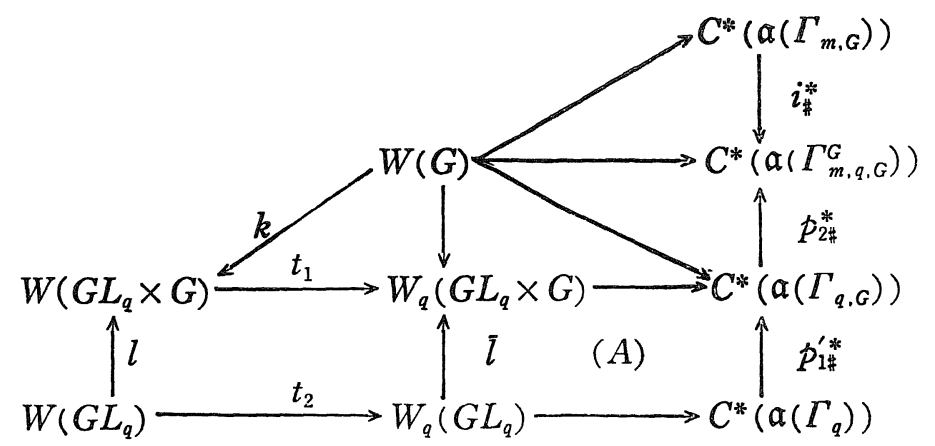

where $W\left(G L_{q}\right)=W\left(G L_{q} \times\{1\}\right)$, and $t_{1}$ and $t_{2}$ are truncating homomorphisms given in Section 2, and $k$ (resp. $l$ ) is an inclusion map defined by $W(G)=1 \otimes W(G) \longrightarrow W\left(G L_{q}\right) \otimes W(G)=W\left(G L_{q} \times G\right)$ (resp. $\left.W\left(G L_{q}\right)=W\left(G L_{q}\right) \otimes 1 \longrightarrow W\left(G L_{q} \times G\right)\right)$. By the definition of the truncations, $G L_{q}$-homomorphism $\bar{l}$ is naturally defined and the square (A) is commutative.

Let us consider the basic cochain subalgebras in the diagrams (8.5) and (8.6) with respect to the products of each maximal compact subgroup and $G$. Before doing that we note some facts. From the definition, the composition map $p_{1 \sharp}^{\prime} \circ p_{2 \sharp}: \mathfrak{a}\left(\Gamma_{m, q, G}^{G}\right) \longrightarrow \mathfrak{a}\left(\Gamma_{q}\right)$ maps right $G$-invariant vector fields on $G$ to the zero vector fields, and $\mathfrak{a}\left(\Gamma_{q}\right)$ is regarded canonically as a Lie subalgebra (of $\mathfrak{a}\left(\Gamma_{m, q, G}^{G}\right)$ ) which is invariant with respect to the adjoint action of $G$. Hence the image of $F_{2 \sharp}^{*} \circ p_{1 \sharp}^{*}$ is contained in $G$ basic cochain subalgebra. The image of $P\left(p_{2}\right) * P\left(p_{1}^{\prime}\right)^{*}$ is also contained in $G$-basic cochain subalgebra, because the $G$-action on $P(\bar{F})$ preserves each fiber of $P\left(p_{1}^{\prime}\right)$. Hence the image of $P\left(p_{2}\right) * \circ\left(p_{1}^{\prime}\right) * \circ P(\pi)^{*}$ is contained in the $G$-basic cochain subalgebra.

First we consider the case that $G$ is connected. In this case any $\mathrm{g}$-basic cochain subalgebra is equal to $G$-basic one. Note that, if a Lie group $L$ acts on a manifold $X$ and the projection $X \longrightarrow X / L$ is a principal $L$-bundle, then $\Omega(X)_{L \text {-basic }}=\Omega(X / L)$. Since the maximal compact subgroup $O(m-q) \times O(q)$ acts on $G$ trivially and $G$ acts on $\mathbb{R}^{m}$ trivially, the actions of $G$ and $O(m-q) \times O(q)$ on $C^{*}\left(\mathfrak{a}\left(\Gamma_{m, q, G}^{G}\right)\right)$ and $\Omega^{*}(P(S))$ etc. are commutative with each other. Then taking 
(maximal compact subgroup) $\times G$-basic cochain subalgebras in the diagrams (8.5) and (8.6), we obtain a commutative diagram:

(8. 7)

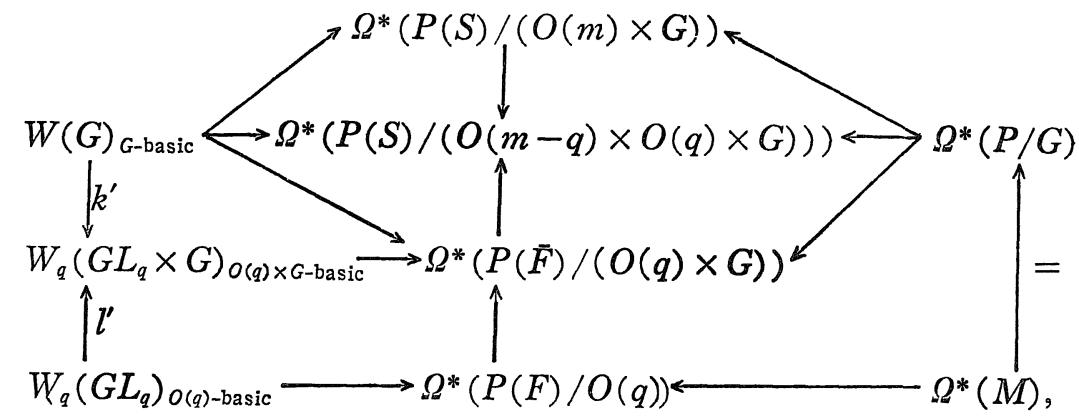

where $k^{\prime}=\left.t_{1} \circ k\right|_{W(G)}$-basic and $l^{\prime}=\left.\bar{l}\right|_{W_{q}\left(G L_{q}\right) O(q) \text {-basic }}$. Since the projection $P^{k}\left(S^{\prime}\right) / O(m) \longrightarrow P$ is not only a homotopy equivalence but also a principal $G$-bundle map, the projection $P^{k}\left(S^{\prime}\right) /(O(m) \times G) \longrightarrow P / G$ $=M$ is a homotopy equivalence. By the same reason other two projections are also homotopy equivalences.

As regards the route through the summit in the diagram (8.7), the following fact is known: Considering the corresponding route in the union of the diagrams (8.5), (8.6), we take $G L_{m} \times G$-basic cochain subalgebras. Then we have the following commutative diagram:

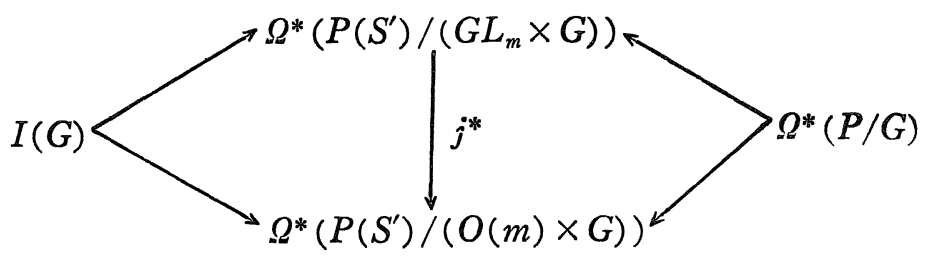

where $j: P\left(S^{\prime}\right) /(O(m) \times G) \longrightarrow P\left(S^{\prime}\right) /\left(G L_{m} \times G\right)$ is the natural projection. Since $\left(\Gamma_{m, G}\right)_{o}^{k} / G L_{m}$ is contractible, the projection $P\left(S^{\prime}\right) /$ $\left(G L_{m} \times G\right) \longrightarrow P / G=M$ induces an isomorphism of cohomology. Hence we have the commutative diagram:

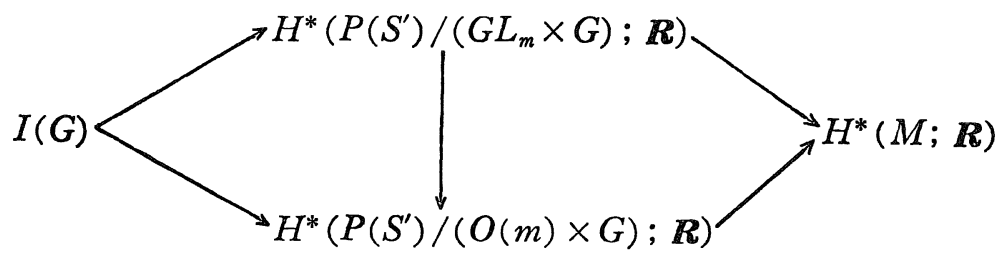


Let $\chi: I(G) \longrightarrow H^{*}(M ; \boldsymbol{R})$ denote the homomorphism defined by this diagram.

Theorem 8.8 ([1, Theorem 11.2]). Let $G$ be a connected Lie group, and $\alpha_{P}$ be the Chern-Weil characteristic homomorphism for a smooth princifal G-bundle $P \longrightarrow M$. Then we have

$$
\chi=\alpha_{P}: I(G) \longrightarrow H^{*}(M ; \mathbb{R}) .
$$

In (8.7) we consider their cohomology algebras. From Example 5. 3, Theorem 7.3 and Theorem 8.8, we have a commutative diagram:

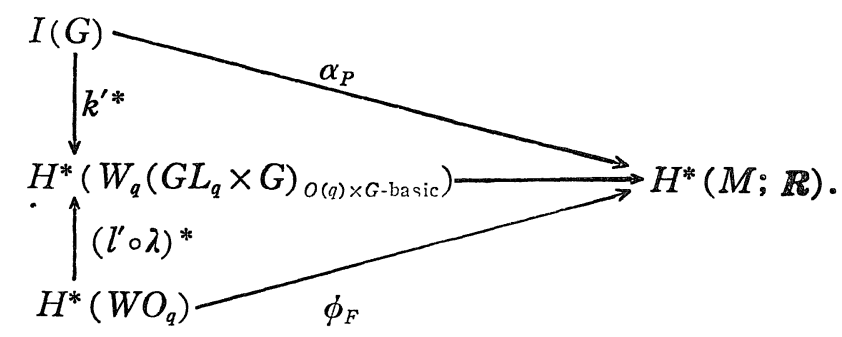

Since $k^{*}(I(G)) \cup\left(l^{\prime} \circ \lambda\right)^{*}\left(\boldsymbol{R}_{q}\left[c_{1}, \cdots, c_{q}\right]\right) \subset \hat{S}\left(\left(\mathfrak{g}_{q} \times \mathfrak{g}\right)^{*}\right)$, for each homogeneous element $\omega \in I(G)$ and each representative cochain $h_{I} c_{J}$ of the Vey basis of $H^{*}\left(W O_{q}\right)$, the product $k^{*}(\omega) \cdot\left(l^{\prime} \circ \lambda\right) *\left(h_{I} c_{J}\right)$ is equal to zero if $\operatorname{deg} \omega+\operatorname{deg} c_{J}>2 q$. Hence using the commutativity of (8. 9), we have

$$
\alpha_{P}(\omega) \cdot \phi_{F}\left(h_{I} c_{J}\right)=0
$$

if $\operatorname{deg} \omega+\operatorname{deg} c_{J}>2 q$.

In order to complete the proof, we must consider the case that $G$ is not connected but has a finite number of connected components. Let $G_{0}$ be the connected component of the unit. Then $G_{0}$ is a normal subgroup of $G$ and the quotient space $P / G_{0}$ is the total space of the principal $G / G_{0}$-bundle $\pi^{\prime}: P / G_{0} \longrightarrow M$. Since $G / G_{0}$ is discrete, the foliation $F$ on $M$ can be lifted on $P / G_{0}$. Let $F^{\prime \prime}$ be the lifted foliation. Then $\pi^{\prime}$ is a morphism of foliation from $F^{\prime \prime}$ to $F$. From Theorem 7.3, we have a commutative diagram:

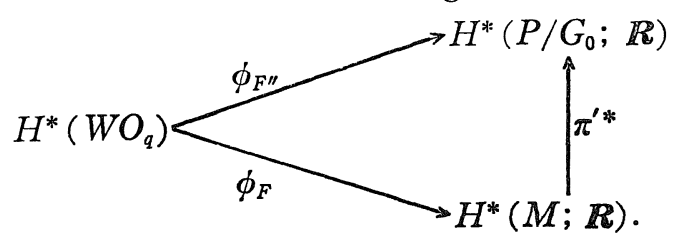


On the other hand $P / G_{0}$ is also a base space of the principal $G_{0}$-bundle $P \longrightarrow P / G_{0}$. By the naturality of the Chern-Weil homomorphism ([7, II, §6.26]), we also have a commutative diagram:

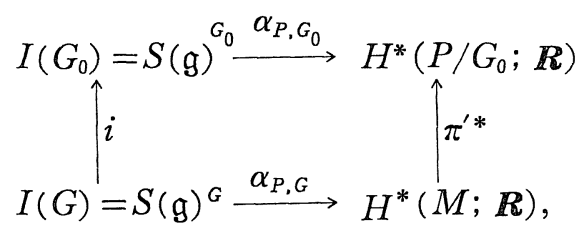

where $i$ is an inclusion map. Take a homogeneous element $\omega$ of $I(G)$ and any $h_{I} c_{J} \in H^{*}\left(W O_{q}\right)$ such that $\operatorname{deg} \omega+\operatorname{deg} c_{J}>2 q$. From (8.10) and (8.11), if there exists a lift of $F$ on $P$, then

$$
\pi^{\prime *}\left(\alpha_{P, G}(\omega) \cdot \phi_{F}\left(h_{I} c_{J}\right)\right)=\alpha_{P, G_{0}} \circ i(\omega) \cdot \phi_{F^{\prime \prime}}\left(h_{I} c_{J}\right)=0,
$$

because the theorem is true when the structure group is connected. Since $\pi^{\prime}$ is also a finite covering map, $\pi^{\prime *}$ is injective. Hence $\alpha_{P, G}(\omega)$ 。 $\phi_{F}\left(h_{I} c_{J}\right)=0$. This completes the proof of Theorem 6. 2 .

\section{References}

[1] Bernshtein, I. and Rozenfel'd, B., Homogeneous spaces of infinite-dimensional Lie algebras and characteristic classes of foliations, Russian Math. Survey, 28 (1973), 107-142.

[2] Bott, R., Lectures on characteristic classes and foliations, Lecture Notes in Math., 279 (1972), 1-80, Springer-Verlag.

[3] Bott, R. and Haefliger, A., On characteristic classes of I'-foliations, Bull. Amer. Math. Soc., 78 (1972), 1039-1044.

[4] Bott, R. and Segal, G., The cohomology of the vector fields on a manifold, Topology, 16 (1977), 285-298.

[5] Gel'fand, I. M. and Fuks, D. B., Cohomology of the Lie algebra of formal vector fields, Izv. Acad. Nauk SSS R, 34 (1970), 327-342.

[6] Godbillon, C., Cohomologies d'algèbres de Lie de champs de vecteurs formels, Séminaire Bourbaki, 421 (1972), 1-19.

[7] Greub, W., Halperin, S. and Vanstone, R., Connection, curvature and cohomology, Vol. II, III, Academic Press, New York, 1976.

[8] Guillemin, V. and Sternberg, S., Deformation theory of pseudogroup structures, Mem. Amer. Math. Soc., 64 (1966).

[9] Hochschild, G. and Serre, J.-P., Cohomology of Lie algebras, Ann. of Math., 57 (1953), 591-603.

[10] Hurder, D., On the secondary classes of foliations with trivial normal bundles, Comment. Math. Helvetici, 56 (1981), 307-326.

[11] Kamber, F. and Tondeur, P., Foliated bundles and characteristic classes, Lecture Notes in Math., 493 (1975), Springer-Verlag.

[12] Molino, P., Classes caractéristiques et obstruction d'Atiyah pour les fibrés principaux feuilletés, C. R. Acad. Sci. Paris, Ser. A-B, 272 (1971), A1376-A1378.

[13] Mostow, M. and Perchik, J., Note on Gel'fand-Fuks cohomology and characteristic 
classes, Proc. of the 11 th Annual Holiday Symposium at New Mexico State University', December 1973, New Mexico State University, 1975.

[14] Rodrigues, A. A. M., On infinite Lie groups, Ann. Inst. Fourier, Grenoble, 31 (1981), 245-274.

[15] Shiga, K., Cohomology of Lie algebras over a manifold, I, Jour. Math. Soc. Japan, 26 (1974), 324-361.

[16] Sullivan, D., Infinitesimal calculations in topology, Publ. Math. I. H. E. S., 47 (1977), 269-331.

[17] Weyl, H., The classical groups, Princeton Univ. Press, Princeton, 1946. 
